\title{
Morphological Signal and Image Processing
}

Petros Maragos

Georgia Institute of Technology
74.1 Introduction

74.2 Morphological Operators for Sets and Signals

Boolean Operators and Threshold Logic $\bullet$ Morphological Set Operators $\bullet$ Morphological Signal Operators and Nonlinear Convolutions

74.3 Median, Rank, and Stack Operators

74.4 Universality of Morphological Operators

74.5 Morphological Operators and Lattice Theory

74.6 Slope Transforms

74.7 Multiscale Morphological Image Analysis Binary Multiscale Morphology via Distance Transforms $\bullet$ Multiresolution Morphology

74.8 Differential Equations for Continuous-Scale Morphology

74.9 Applications to Image Processing and Vision Noise Suppression • Feature Extraction • Shape Representation via Skeleton Transforms $\bullet$ Shape Thinning $\bullet$ Size Distributions

- Fractals • Image Segmentation

74.10 Conclusions

Acknowledgment

References

\subsection{Introduction}

This chapter provides a brief introduction to the theory of morphological signal processing and its applications to image analysis and nonlinear filtering. By "morphological signal processing" we mean a broad and coherent collection of theoretical concepts, mathematical tools for signal analysis, nonlinear signal operators, design methodologies, and applications systems that are based on or related to mathematical morphology (MM), a set- and lattice-theoretic methodology for image analysis. MM aims at quantitatively describing the geometrical structure of image objects. Its mathematical origins stem from set theory, lattice algebra, convex analysis, and integral and stochastic geometry. It was initiated mainly by Matheron [42] and Serra [58] in the 1960s. Some of its early signal operations are also found in the work of other researchers who used cellular automata and Boolean/threshold logic to analyze binary image data in the 1950s and 1960s, as surveyed in [49, 54]. MM has formalized these earlier operations and has also added numerous new concepts and image operations. In the 1970s it was extended to gray-level images [22, 45, 58, 62]. Originally MM was applied to analyzing 
images from geological or biological specimens. However, its rich theoretical framework, algorithmic efficiency, easy implementability on special hardware, and suitability for many shape-oriented problems have propelled its widespread diffusion and adoption by many academic and industry groups in many countries as one among the dominant image analysis methodologies. Many of these research groups have also extended the theory and applications of MM. As a result, MM nowadays offers many theoretical and algorithmic tools to and inspires new directions in many research areas from the fields of signal processing, image processing and machine vision, and pattern recognition.

As the name 'morphology' implies (study/analysis of shape/form), morphological signal processing can quantify the shape, size, and other aspects of the geometrical structure of signals viewed as image objects, in a rigorous way that also agrees with human intuition and perception. In contrast, the traditional tools of linear systems and Fourier analysis are of limited or no use for solving geometrybased problems in image processing because they do not directly address the fundamental issues of how to quantify shape, size, or other geometrical structures in signals and may distort important geometrical features in images. Thus, morphological systems are more suitable than linear systems for shape analysis. Further, they offer simple and efficient solutions to other nonlinear problems, such as non-Gaussian noise suppression or envelope estimation. They are also closely related to another class of nonlinear systems, the median, rank, and stack operators, which also outperform linear systems in non-Gaussian noise suppression and in signal enhancement with geometric constraints. Actually, rank and stack operators can be represented in terms of elementary morphological operators. All of the above, coupled with the rich mathematical background of mathematical morphology, make morphological signal processing a rigorous and efficient framework to study and solve many problems in image analysis and nonlinear filtering.

\subsection{Morphological Operators for Sets and Signals}

\subsubsection{Boolean Operators and Threshold Logic}

Early works in the fields of visual pattern recognition and cellular automata dealt with analysis of binary digital images using local neighborhood operations of the Boolean type. For example, given a sampled ${ }^{1}$ binary image signal $f[x]$ with values 1 for the image foreground and 0 for the background, typical signal transformations involving a neighborhood of $n$ samples whose indices are arranged in a window set $W=\left\{y_{1}, y_{2}, \ldots, y_{n}\right\}$ would be

$$
\psi_{b}(f)[x]=b\left(f\left[x-y_{1}\right], \ldots, f\left[x-y_{n}\right]\right)
$$

where $b\left(v_{1}, \ldots, v_{n}\right)$ is a Boolean function of $n$ variables. The mapping $f \mapsto \psi_{b}(f)$ is a nonlinear system, called a Boolean operator. By varying the Boolean function $b$, a large variety of Boolean operators can be obtained; see Table 74.1 where $W=\{-1,0,1\}$. For example, choosing a Boolean AND for $b$ would shrink the input image foreground, whereas a Boolean OR would expand it.

Two alternative implementations and views of these Boolean operations are (1) thresholded convolutions, where a binary input is linearly convolved with an $n$-point mask of ones and then the output is thresholded at 1 or $n$ to produce the Boolean OR or AND, respectively, and (2) min / max operations, where the moving local minima and maxima of the binary input signal produce the same output as Boolean AND/OR, respectively. In the thresholded convolution interpretation, thresholding at an intermediate level $r$ between 1 and $n$ produces a binary rank operation of the binary input data (inside the moving window). For example, if $r=(n+1) / 2$, we obtain the binary median filter whose

\footnotetext{
${ }^{1}$ Signals of a continuous variable $x \in \mathbb{R}^{d}$ are usually denoted by $f(x)$, whereas for signals with discrete variable $x \in \mathbb{Z}^{d}$ we write $f[x]$.
} 
TABLE 74.1 Discrete Set Operators and Their

\begin{tabular}{cc} 
Generating Boolean Function \\
\hline Set Operator $\Psi(X), X \subseteq \mathbb{Z}$ & Boolean function $b\left(v_{1}, v_{2}, v_{3}\right)$ \\
\hline Erosion: $X \ominus\{-1,0,1\}$ & $v_{1} v_{2} v_{3}$ \\
Dilation: $X \oplus\{-1,0,1\}$ & $v_{1}+v_{2}+v_{3}$ \\
Median: $X \square_{2}\{-1,0,1\}$ & $v_{1} v_{2}+v_{1} v_{3}+v_{2} v_{3}$ \\
Hit-Miss: $X \otimes(\{-1,1\},\{0\})$ & $v_{1} v_{2} v_{3}$ \\
Opening: $X \circ\{0,1\}$ & $v_{1} v_{2}+v_{2} v_{3}$ \\
Closing: $X \bullet\{0,1\}$ & $v_{2}+v_{1} v_{3}$ \\
\hline
\end{tabular}

Boolean function expresses the majority voting logic; see the third example of Table 74.1. Of course, numerous other Boolean operators are possible, since there are $2^{2^{n}}$ possible Boolean functions of $n$ variables. The main applications of such Boolean signal operations have been in biomedical image processing, character recognition, object detection, and general 2D shape analysis. Detailed accounts and more references of these approaches and applications can be found in [49,54].

\subsubsection{Morphological Set Operators}

Among the new important conceptual leaps offered by mathematical morphology was to use sets to represent binary image signals and set operations to represent binary image transformations. Specifically, given a binary image, let its foreground be represented by the set $X$ and its background by the set complement $X^{c}$. The Boolean OR transformation of $X$ by a (window) set $B$ (local neighborhood of pixels) is mathematically equivalent to the Minkowski set addition $\oplus$, also called dilation, of $X$ by $B$ :

$$
X \oplus B \equiv\{x+y: x \in X, y \in B\}=\bigcup_{y \in B} X_{+y}
$$

where $X_{+y} \equiv\{x+y: x \in X\}$ is the translation of $X$ along the vector $y$. Likewise, if $B^{r} \equiv\{x:-x \in$ $B$ \} denotes the reflection of $B$ with respect to the axes' origin, the Boolean AND transformation of $X$ by the reflected $B$ is equivalent to the Minkowski set subtraction [24] $\ominus$, also called erosion, of $X$ or $B$ :

$$
X \ominus B \equiv\left\{x: B_{+x} \subseteq X\right\}=\bigcap_{y \in B} X_{-y}
$$

In applications, $B$ is usually called a structuring element and has a simple geometrical shape and a size smaller than the image set $X$. As shown in Fig. 74.1, erosion shrinks the original set, whereas dilation expands it.

The erosion (74.2) can also be viewed as Boolean template matching since it gives the center points at which the shifted structuring elements fits inside the image foreground. If we now consider a set $A$ probing the image foreground set $X$ and another set $B$ probing the background $X^{c}$, the set of points at which the shifted pair $(A, B)$ fits inside the images is the hit-miss transformation of $X$ by $(A, B)$ :

$$
X \otimes(A, B) \equiv\left\{x: A_{+x} \subseteq X, B_{+x} \subseteq X^{c}\right\}
$$

In the discrete case, this can be represented by a Boolean product function whose uncomplemented (complemented) variables correspond to points of $A(B)$; see Table 74.1. It has been used extensively for binary feature detection [58] and especially in document image processing [8,9].

Dilating an eroded set by the same structuring element in general does not recover the original set but only a part of it, its opening. Performing the same series of operations to the set complement yields a set containing the original, its closing. Thus, cascading erosion and dilation gives rise to two new operations, the opening $X \circ B \equiv(X \ominus B) \oplus B$ and the closing $X \bullet B \equiv(X \oplus B) \ominus B$ of $X$ by $B$. As shown in Fig. 74.1, the opening suppresses the sharp capes and cuts the narrow isthmuses of $X$, whereas the closing fills in the thin gulfs and small holes. Thus, if the structuring element $B$ 

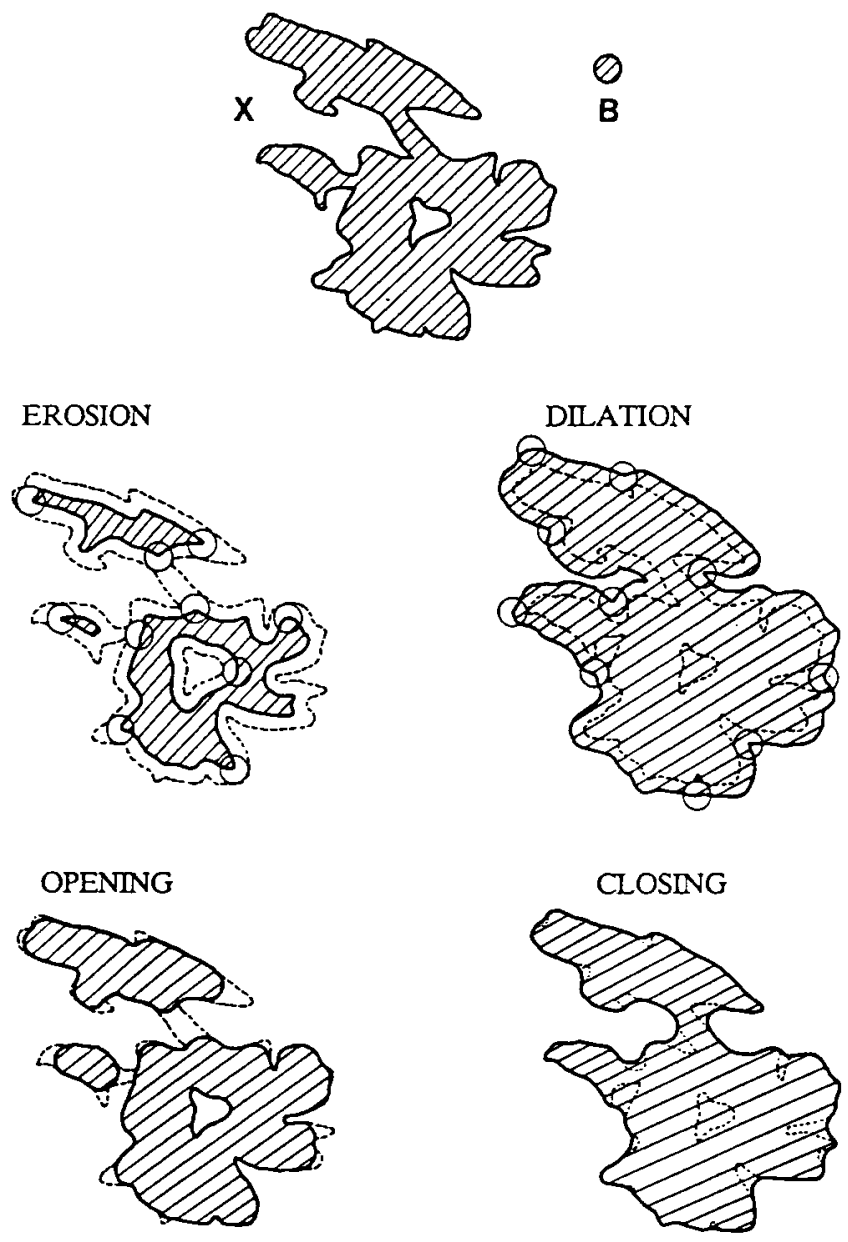

FIGURE 74.1: Erosion, dilation, opening, and closing of $X$ (binary image of an island) by a disk $B$ centered at the origin. The shaded areas correspond to the interior of the sets, the dark solid curve to the boundary of the transformed sets, and the dashed curve to the boundary of the original set $X$.

has a regular shape, both opening and closing can be thought of as nonlinear filters which smooth the contours of the input signal.

These set operations make mathematical morphology more general than previous approaches because it unifies and systematizes all previous digital and analog binary image operations, mathematically rigorous and notationally elegant since it is based on set theory, and intuitive since the set formalism is easily connected to mathematical logic. Further, the basic morphological set operators directly relate to the shape and size of binary images in a way that has many common points with human perception about geometry and spatial reasoning.

\subsubsection{Morphological Signal Operators and Nonlinear Convolutions}

In the 1970s, morphological operators were extended from binary to gray-level images and realvalued signals. Going from sets to functions was made possible by using set representations of signals and transforming these input sets via morphological set operations. Thus, consider a signal $f(x)$ 
defined on the $d$-dimensional continuous or discrete domain $\mathbb{D}=\mathbb{R}^{d}$ or $\mathbb{Z}^{d}$ and assuming values in $\overline{\mathbb{R}}=\mathbb{R} \cup\{-\infty, \infty\}$. Thresholding the signal at all amplitude values $v$ produces an ensemble of threshold binary signals

$$
\theta_{v}(f)(x) \equiv 1 \text { if } f(x) \geq v, \text { and } 0 \text { else, }
$$

represented by the threshold sets [58]

$$
\Theta_{v}(f) \equiv\{x \in \mathbb{D}: f(x) \geq v\}, \quad-\infty<v<+\infty
$$

The signal can be exactly reconstructed from all its thresholded versions since

$$
f(x)=\sup \left\{v \in \mathbb{R}: x \in \Theta_{v}(f)\right\}=\sup \left\{v \in \mathbb{R}: \theta_{v}(f)(x)=1\right\}
$$

Transforming each threshold set by a set operator $\Psi$ and viewing the transformed sets as threshold sets of a new signal creates a flat signal operator $\psi$ whose output is

$$
\psi(f)(x)=\sup \left\{v \in \mathbb{R}: x \in \Psi\left[\Theta_{v}(f)\right]\right\}
$$

Using set dilation and erosion in place of $\Psi$, the above procedure creates the two most elementary morphological signal operators: the dilation and erosion of a signal $f(x)$ by a set $B$ :

$$
\begin{aligned}
& (f \oplus B)(x)=\bigvee_{y \in B} f(x-y) \\
& (f \ominus B)(x) \equiv \bigwedge_{y \in B} f(x+y)
\end{aligned}
$$

where $\bigvee$ denotes supremum (or maximum for finite $B$ ) and $\bigwedge$ denotes infimum (or minimum for finite $B$ ). These gray-level morphological operations can also be created from their binary counterparts using concepts from fuzzy sets where set union and intersection becomes maximum and minimum on gray-level images [22, 45]. As Fig. 74.2 shows, flat erosion (dilation) of a function $f$ by a small convex set $B$ reduces (increases) the peaks (valleys) and enlarges the minima (maxima) of the function. The flat opening $f \circ B=(f \ominus B) \oplus B$ of $f$ by $B$ smooths the graph of $f$ from below by cutting down its peaks, whereas the closing $f \bullet B=(f \oplus B) \ominus B$ smoothes it from above by filling up its valleys.

More general morphological operators for gray-level 2D image signals $f(x)$ can be created [62] by representing the surface of $f$ and all the points underneath by a 3D set $U(f)=\{(x, v): v \leq f(x)\}$, called its umbra; then dilating or eroding $U(f)$ by the umbra of another signal $g$ yields the umbras of two new signals, the dilation or erosion of $f$ by $g$, which can be computed directly by the formulae:

$$
\begin{aligned}
& (f \oplus g)(x) \quad \equiv \bigvee_{y \in \mathbb{D}} f(x-y)+g(y) \\
& (f \ominus g)(x) \equiv \bigwedge_{y \in \mathbb{D}} f(x+y)-g(y)
\end{aligned}
$$

and two supplemental rules for adding and subtracting with infinities: $r \pm s=-\infty$ if $r=-\infty$ or $s=-\infty$, and $+\infty-r=+\infty$ if $r \in \mathbb{R} \cup\{+\infty\}$. These two signal transformations are nonlinear and translation-invariant. Their computational structure closely resembles that of a linear convolution $(f * g)[x]=\sum_{y} f[x-y] g[y]$ if we correspond the sum of products to the supremum of sums in the dilation. Actually, in the areas of convex analysis [50] and optimization [6], the operation (74.10) has been known as the supremal convolution. Similarly, replacing $-g(-x)$ with $g(x)$ in the erosion (74.11) yields the infimal convolution

$$
(f \square g)(x) \equiv \bigwedge_{y \in \mathbb{D}} f(x-y)+g(y)
$$



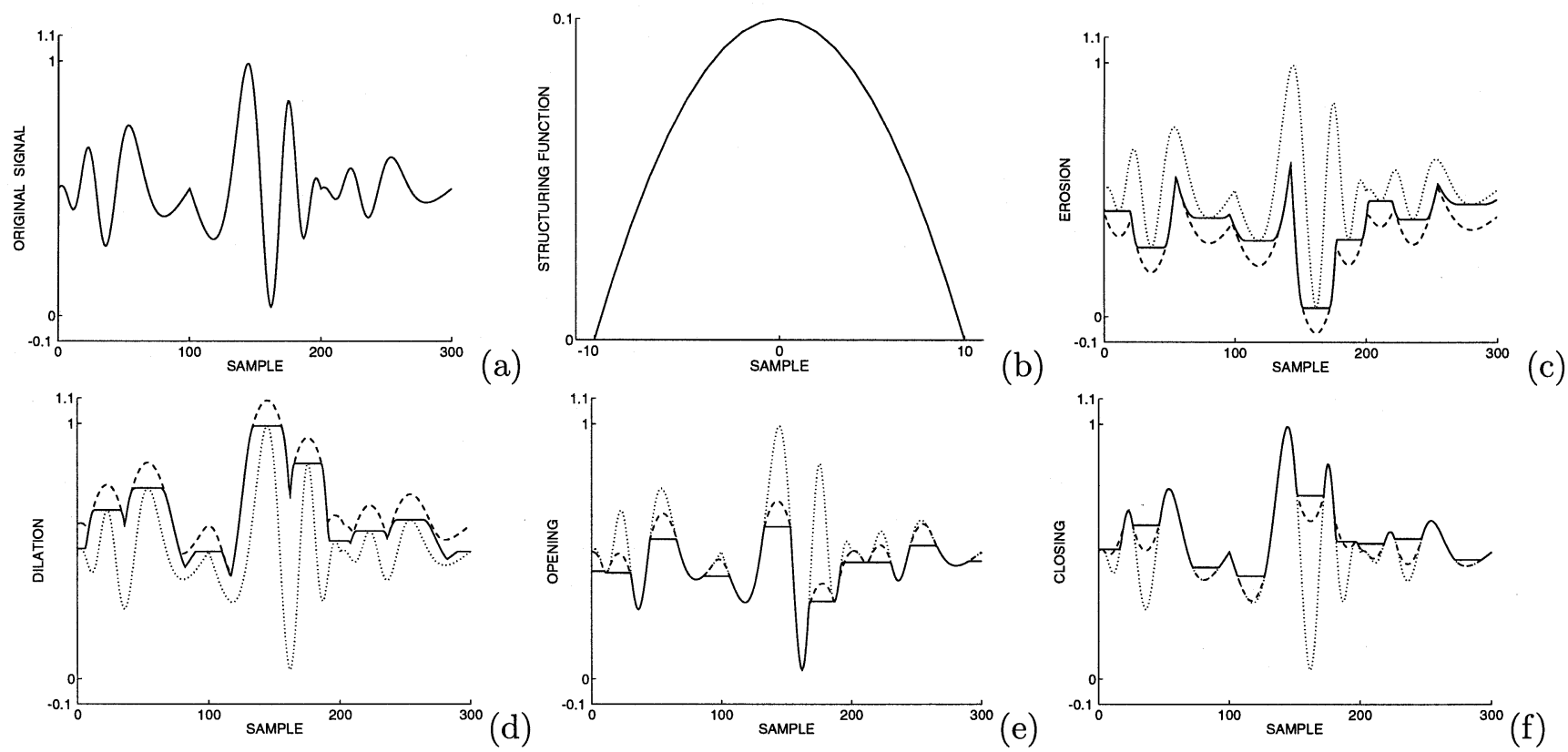

(b)

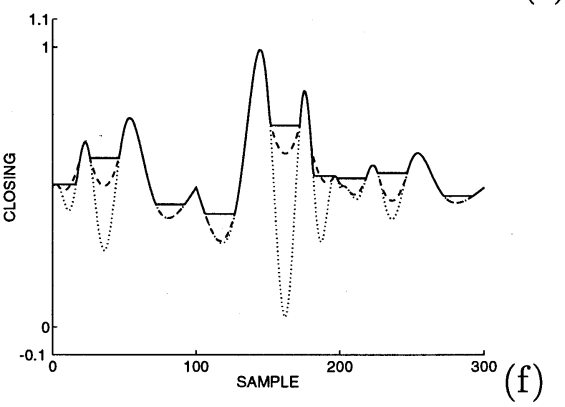

FIGURE 74.2: (a) Original signal $f$. (b) Structuring function $g$ (a parabolic pulse). (c) Erosion $f \ominus g$ with dashed line and flat erosion $f \ominus B$ with solid line, where the set $B=\{x \in \mathbb{Z}:|x| \leq 10\}$ is the support of $g$. Dotted line shows original signal $f$. (d) Dilation $f \oplus g$ (dashed line) and flat dilation $f \oplus B$ (solid line). (e) Opening $f \circ g$ (dashed line) and flat opening $f \circ B$ (solid line). (f) Closing $f \bullet g$ (dashed line) and flat closing $f \bullet B$ (solid line). 
The nonlinearity of $\oplus$ and $\ominus$ causes some differences between these signal operations and the linear convolutions. A major difference is that serial or parallel interconnections of systems represented by linear convolutions are equivalent to an overall linear convolution, whereas interconnections of dilations and erosions lead to entirely different nonlinear systems. Thus, there is an infinite variety of nonlinear operators created by cascading dilations and erosions or by interconnecting them in parallel via max / min or addition. Two such useful examples are the opening $\circ$ and closing $\bullet$

$$
\begin{aligned}
& f \circ g \equiv(f \ominus g) \oplus g \\
& f \bullet g \equiv(f \oplus g) \ominus g
\end{aligned}
$$

which act as nonlinear smoothers.

Figure 74.2 shows that the four basic morphological transformations of a 1D signal $f$ by a concave even function $g$ with a compact support $B$ have similar effects as the corresponding flat transformations by the set $B$. Among the few differences, the erosion (dilation) of $f$ by $g$ subtracts from (adds to) $f$ the values of the moving template $g$ during the decrease (increase) of signal peaks (valleys) and the broadening of the local signal minima (maxima) that would incur during erosion (dilation) by $B$. Similarly, the opening (closing) of $f$ by $g$ cuts the peaks (fills up the valleys) inside which no translated version of $g(-g)$ can fit and replaces these eliminated peaks (valleys) by replicas of $g(-g)$. In contrast, the flat opening or closing by $B$ only cuts the peaks or fills valleys and creates flat plateaus in the output.

The four above morphological operators of dilation, erosion, opening, and closing have a rich collection of algebraic properties, some of which are listed in Tables 74.2 and 74.3, which endow them with a broad range of applications, make them rigorous, and lead to a variety of efficient serial or parallel implementations.

TABLE 74.2 Definitions of Operator Properties

\begin{tabular}{ccc}
\hline Property & Set operator $\Psi$ & Signal operator $\psi$ \\
\hline Translation-Invar. & $\Psi(X+y)=\Psi(X)_{+y}$ & $\psi[f(x-y)+c]=c+\psi(f)(x-y)$ \\
Shift-Invariant & $\Psi(X+y)=\Psi(X)_{+y}$ & $\psi[f(x-y)]=\psi(f)(x-y)$ \\
Increasing & $X \subseteq Y \rightleftharpoons \Psi(X) \subseteq \Psi(Y)$ & $f \leq g \Longrightarrow \psi(f) \leq \psi(g)$ \\
Extensive & $X \subseteq \Psi(X)$ & $f \leq \psi(f)$ \\
Anti-extensive & $\Psi(X) \subseteq X$ & $\psi(f) \leq f$ \\
Idempotent & $\Psi(\Psi(X))=\Psi(X)$ & $\psi(\psi(f))=\psi(f)$ \\
\hline
\end{tabular}

TABLE 74.3 Properties of Basic Morphological Signal Operators

\begin{tabular}{ccccc}
\hline Property & Dilation & Erosion & Opening & Closing \\
\hline Duality & $f \oplus g=-\left[(-f) \ominus g^{r}\right]$ & & $f \circ g=-\left[(-f) \bullet g^{r}\right]$ & \\
Distributivity & $\left(\vee_{i} f_{i}\right) \oplus g=\vee_{i} f_{i} \oplus g$ & $\left(\wedge_{i} f_{i}\right) \ominus g=\wedge_{i} f_{i} \ominus g$ & No & No \\
Composition & $(f \oplus g) \oplus h=f \oplus(g \oplus h)$ & $(f \ominus g) \ominus h=f \ominus(g \oplus h)$ & & No \\
Extensive & Yes if $g(0) \geq 0$ & No & Yes & No \\
Anti-Extensive & No & Yes if $g(0) \geq 0$ & No & No \\
Commutative & $f \oplus g=g \oplus f$ & No & Yes & Yes \\
Increasing & Yes & Yes & Yes & Yes \\
Translation-Invar. & Yes & Yes & Yes & Yes \\
Idempotent & No & No & & \\
\hline
\end{tabular}




\subsection{Median, Rank, and Stack Operators}

Flat erosion and dilation of a discrete-domain signal $f[x]$ by a finite window $W=\left\{y_{1}, \ldots, y_{n}\right\} \subseteq \mathbb{Z}^{d}$ is a moving local minimum or maximum. Replacing min / max with a more general rank leads to rank operators. At each location $x \in \mathbb{Z}^{d}$, sorting the signal values within the reflected and shifted $n$-point window $\left(W^{r}\right)_{+x}$ in decreasing order and picking the $p$ th largest value, $p=1,2, \ldots, n=$ card $(W)$, yields the output signal from the $p$ th rank operator:

$$
\left(f \square_{p} W\right)[x] \equiv p \text { th } \operatorname{rank} \text { of }\left(f\left[x-y_{1}\right], \ldots, f\left[x-y_{n}\right]\right)
$$

For odd $n$ and $p=(n+1) / 2$ we obtain the median operator. If the input signal is binary, the output is also binary since sorting preserves a signal's range. Representing the input binary signal with a set $S \subseteq \mathbb{Z}^{d}$, the output set produced by the $p$ th rank set operators is

$$
S \square_{p} W \equiv\left\{x: \operatorname{card}\left(\left(W^{r}\right)_{+x} \cap S\right) \geq p\right\}
$$

Thus, computing the output from a set rank operator involves only counting of points and no sorting.

All rank operators commute with thresholding [21, 27, 41, 45, 58, 65]; i.e.,

$$
\Theta_{v}\left[f \square_{p} W\right]=\left[\Theta_{v}(f)\right] \square_{p} W, \forall v, \forall p .
$$

This property is also shared by all morphological operators that are finite compositions or max$\mathrm{ima} / \mathrm{minima}$ of flat dilations and erosions, e.g., openings and closings, by finite structuring elements. All such signal operators $\psi$ that have a corresponding set operator $\Psi$ and commute with thresholding can be alternatively implemented via threshold superposition [41, 58] as in (74.7). Namely, to transform a multilevel signal $f$ by $\psi$ is equivalent to decomposing $f$ into all its threshold sets, transforming each set by the corresponding set operator $\Psi$, and reconstructing the output signal $\psi(f)$ via its thresholded versions. This allows us to study all rank operators and their cascade or parallel (using $\vee, \wedge$ ) combinations by focusing on their corresponding binary operators. Such representations are much simpler to analyze and they suggest alternative implementations that do not involve numeric comparisons or sorting.

Binary rank operators and all other binary discrete translation-invariant finite-window operators can be described by their generating Boolean function; see Table 74.1. Thus, in synthesizing discrete multilevel signal operators from their binary countparts via threshold superposition all that is needed is knowledge of this Boolean function. Specifically, transforming all the threshold binary signals $\theta_{v}(f)[x]$ of an input signal $f[x]$ with an increasing Boolean function $b\left(u_{1}, \ldots, u_{n}\right)$ (i.e., containing no complemented variables) in place of the set operator $\Psi$ in (74.7) creates a large variety of nonlinear signal operators via threshold superposition, called stack filters [41, 70]

$$
\phi_{b}(f)[x] \equiv \sup \left\{v: b\left(\theta_{v}(f)\left[x-y_{1}\right], \ldots, \theta_{v}(f)\left[x-y_{n}\right]\right)=1\right\}
$$

For example, $\phi_{b}$ becomes the $p$ th rank operator if $b$ is equal to the sum $\left(\begin{array}{l}n \\ p\end{array}\right)$ product terms where each contains one distinct $p$-point subset from the $n$ variables. In general, the use of Boolean functions facilitates the design of such discrete flat operators with determinable structural properties. Since each increasing Boolean function can be uniquely represented by an irreducible sum (product) of product (sum) terms, and each product (sum) term corresponds to an erosion (dilation), each stack filter can be represented as a finite maximum (minimum) of flat erosions (dilations) [41].

\subsection{Universality of Morphological Operators}

Dilations or erosions, the basic nonlinear convolutions of morphological signal processing, can be combined in many ways to create more complex morphological operators that can solve a broad 
variety of problems in image analysis and nonlinear filtering. In addition, they can be implemented using simple and fast software or hardware; examples include various digital [58, 61] and analog, i.e., optical or hybrid optical-electronic implementations [46, 63]. Their wide applicability and ease of implementation poses the question which signal processing systems can be represented by using dilations and erosions as the basic building blocks. Toward this goal, a theory was introduced in $[33,34]$ that represents a broad class of nonlinear and linear operators as a minimal combination of erosions or dilations. Here we summarize the main results of this theory, in a simplified way, restricting our discussion only to signals with discrete domain $\mathbb{D}=\mathbb{Z}^{d}$.

Consider a translation-invariant set operator $\Psi$ on the class $\mathcal{P}(\mathbb{D})$ of all subsets of $\mathbb{D}$. Any such $\Psi$ is uniquely characterized by its kernel that is defined [42] as the subclass $\operatorname{Ker}(\Psi) \equiv\{X \in \mathcal{P}(\mathbb{D}): 0 \in$ $\Psi(X)$ \} of input sets, where 0 is the origin of $\mathbb{D}$. If $\Psi$ is also increasing, then it can be represented [42] as the union of erosions by its kernel sets and as the intersection of dilations by the reflected kernel sets of its dual operator $\Psi^{d}(X) \equiv\left[\Psi\left(X^{c}\right)\right]^{c}$. This kernel representation can be extended to signal operators $\psi$ on the class Fun $(\mathbb{D}, \overline{\mathbb{R}})$ of signals with domain $\mathbb{D}$ and range $\overline{\mathbb{R}}$. The kernel of $\psi$ is defined as the subclass $\operatorname{Ker}(\psi)=\{f \in \operatorname{Fun}(\mathbb{D}, \overline{\mathbb{R}}):[\psi(f)](0) \geq 0\}$ of input signals. If $\psi$ is translationinvariant and increasing, then it can be represented $[33,34]$ as the pointwise supremum of erosions by its kernel functions, and as the infimum of dilations by the reflected kernel functions of its dual operator $\psi^{d}(f) \equiv-\psi(-f)$.

The two previous kernel representations require an infinite number of erosions or dilations to represent a given operator because the kernel contains an infinite number of elements. However, we can find more efficient (requiring less erosions) representations by using only a substructure of the kernel, its basis. The basis Bas(.) of a set (signal) operator is defined [33, 34] as the collection of kernel elements that are minimal with respect to the ordering $\subseteq(\leq)$.

If a translation-invariant increasing set operator $\Psi$ is also upper semicontinuous, i.e., obeys a monotonic continuity where $\Psi\left(\bigcap_{n} X_{n}\right)=\bigcap_{n} \Psi\left(X_{n}\right)$ for any decreasing set sequence $X_{n}$, then $\Psi$ has a nonempty basis and can be represented via erosions only by its basis sets. If the dual $\Psi^{d}$ is also upper semicontinuous, then its basis sets provide an alternative representation of $\Psi$ via dilations:

$$
\Psi(X)=\bigcup_{A \in \operatorname{Bas}(\Psi)} X \ominus A=\bigcap_{B \in \operatorname{Bas}\left(\Psi^{d}\right)} X \oplus B^{r}
$$

Similarly, any signal operator $\psi$ that is translation-invariant, increasing, and upper semicontinuous (i.e., $\psi\left(\wedge_{n} f_{n}\right)=\wedge_{n} \psi\left(f_{n}\right)$ for any decreasing function sequence $\left.f_{n}\right)$ can be represented as the supremum of erosions by its basis functions, and (if $\psi^{d}$ is upper semicontinuous) as the infimum of dilations by the reflected basis functions of its dual operators:

$$
\psi(f)=\bigvee_{g \in \operatorname{Bas}(\psi)} f \ominus g=\bigwedge_{h \in \operatorname{Bas}\left(\psi^{d}\right)} f \oplus h^{r}
$$

where $h^{r}(x) \equiv h(-x)$. Finally, if $\phi$ is a flat signal operator as in (74.7) that is translation-invariant and commutes with thresholding, then $\phi$ can be represented as a supremum of erosions by the basis sets of its corresponding set operator $\Phi$ :

$$
\phi(f)=\bigvee_{A \in \operatorname{Bas}(\Phi)} f \ominus A=\bigwedge_{B \in \operatorname{Bas}\left(\Phi^{d}\right)} f \oplus B^{r}
$$

While all the above representations express translation-invariant increasing operators via erosions or dilations, operators that are not necessarily increasing can be represented [4] via operations closely related to hit-miss transformations.

Representing operators that satisfy a few general properties in terms of elementary morphological operations can be applied to more complex morphological systems and various other filters such as linear rank, hybrid linear/rank, and stack filters, as the following examples illustrate. 
EXAMPLE 74.1: Morphological Filters

All systems made up of serial or sup/inf combinations of erosions, dilations, opening, and closings admit a basis, which is finite if the system's local definition depends on a finite window. For example, the set opening $\Phi(X)=X \circ A$ has as a basis the set collection $\operatorname{Bas}(\Phi)=\left\{A_{-a}: a \in A\right\}$. Consider now 1D discrete-domain signals and let $A=\{-1,0,1\}$. Then, the basis of $\Phi$ has 3 sets: $G_{1}=$ $A_{-1}, G_{2}=A, G_{3}=A_{+1}$. The basis of the dual operator $\Phi^{d}(X)=X \bullet A$ has 4 sets: $H_{1}=$ $\{0\}, H_{2}=\{-2,1\}, H_{3}=\{-1,2\}, H_{4}=\{-1,1\}$. The flat signal operator corresponding to $\Phi$ is the opening $\phi(f)=f \circ A$. Thus, from (74.21), the signal opening can also be realized as a $\max (\min )$ of local minima (maxima):

$$
(f \circ A)[x]=\bigvee_{i=1}^{3}\left\{\bigwedge_{y \in G_{i}} f[x+y]\right\}=\bigwedge_{k=1}^{4}\left\{\bigvee_{y \in H_{k}} f[x+y]\right\} .
$$

\section{EXAMPLE 74.2: Linear Filters}

A linear shift-invariant filter is translation-invariant and increasing (see Table 74.2 for definitions) if its impulse response is everywhere nonnegative and has area equal to one. Consider the 2-point FIR filter $\psi(f)[x]=a f[x]+(1-a) f[x-1]$, where $0<a<1$. The basis of $\psi$ consists of all functions $g[x]$ with $g[0]=r \in \mathbb{R}, g[-1]=-\operatorname{ar} /(1-a)$, and $g[x]=-\infty$ for $x \neq 0,-1$. Then (74.20) yields

$$
a f[x]+(1-a) f[x-1]=\bigvee_{r \in \mathbb{R}}\left[\min \left\{f[x]-r, f[x-1]+\frac{a r}{1-a}\right\}\right],
$$

which expresses a linear convolution as a supremum of erosions. FIR linear filters have an infinite basis, which is a finite-dimensional vector space.

\section{EXAMPLE 74.3: Median Filters}

All rank operators have a finite basis; hence, they can be expressed as a finite max-of-erosions or min-of-dilations. Further, they commute with thresholding, which allows us to focus only on their binary versions. For example, the set median by the window $W=\{-1,0,1\}$ has 3 basis sets: $\{-1,0\},\{-1,1\}$, and $\{0,1\}$. Hence, (74.21) yields

$$
\operatorname{median}(f[x-1], f[x], f[x+1])=\max \left\{\begin{array}{l}
\min (f[x-1], f[x]), \\
\min [f(x-1), f(x+1)], \\
\min [f(x), f(x+1)]
\end{array}\right\} .
$$

\section{EXAMPLE 74.4: Stack Filters}

Stack filters (74.18) are discrete translation-invariant flat operators $\phi_{b}$, locally defined on a finite window $W$, and are generated by a increasing Boolean function $b\left(v_{1}, \ldots, v_{n}\right)$, where $n=\operatorname{card}(W)$. This function corresponds to a translation-invariant increasing set operator $\Phi$. For example, consider 1D signals, let $W=\{-2,-1,0,1,2\}$ and

$$
b\left(v_{1}, \ldots, v_{5}\right)=v_{1} v_{2} v_{3}+v_{2} v_{3} v_{4}+v_{3} v_{4} v_{5}=v_{3}\left(v_{1}+v_{4}\right)\left(v_{2}+v_{4}\right)\left(v_{2}+v_{5}\right) \text {. }
$$


This function generates via threshold superposition the flat opening $\phi_{b}(f)=f \circ A, A=\{-1,0,1\}$, of (74.22). There is one-to-one correspondence between the three prime implicants of $b$ and the erosions (local min) by the three basis sets of $\Phi$, as well as between the four prime implicates of $\beta$ and the dilations (local max) by the four basis sets of the dual $\Phi^{d}$. In general, given $b, \Phi$ or $\phi_{b}$ is found by replacing Boolean AND/OR with set $\cap / \cup$ or with min / max, respectively. Conversely, given $\phi_{b}$, we can find its generating Boolean function from the basis of its set operator (or directly from its $\max / \mathrm{min}$ representation if available) [41].

The above examples show the power of the general representation theorems. An interesting applications of these results is the design of morphological systems via their basis [5, 20, 31]. Given the wide applicability of erosions/dilations, their parallelism, and their simple implementations, the previous theorems theoretically support a general purpose vision (software or hardware) module that can perform erosions/dilations, based on which numerous other complex image operations can be built.

\subsection{Morphological Operators and Lattice Theory}

In the late 1980s and 1990s a new and more general formalization of morphological operators was introduced [59, chaps.1,5-8], [26, 51, 52], which views them as operators on complete lattices. A complete lattice is a set $\mathcal{L}$ equipped with a partial ordering $\leq$ such that $(\mathcal{L}, \leq)$ has the algebraic structure of a partially ordered set (poset) where the supremum and infimum of any of its subsets exist in $\mathcal{L}$. For any subset $\mathcal{K} \subseteq \mathcal{L}$, its supremum $\vee \mathcal{K}$ and infimum $\wedge \mathcal{K}$ are defined as the lowest (with respect to $\leq$ ) upper bound and greatest lower bound of $\mathcal{K}$, respectively. The two main examples of complete lattices used in morphological processing are: (1) the set space $\mathcal{P}(\mathbb{D})$ where the $\vee / \wedge$ lattice operations are the set union/intersection, and (2) the signal space Fun $(\mathbb{D}, \overline{\mathbb{R}})$ where the $\vee / \wedge$ lattice operations are the supremum/infimum of sets of real numbers. Increasing operators on $\mathcal{L}$ are of great importance because they preserve the partial ordering, and among them four fundamental examples are:

$$
\begin{aligned}
\delta \text { is dilation } & \Longleftrightarrow \delta\left(\bigvee_{i \in I} f_{i}\right)=\bigvee_{i \in I} \delta\left(f_{i}\right) \\
\varepsilon \text { is erosion } & \Longleftrightarrow \varepsilon\left(\bigwedge_{i \in I} f_{i}\right)=\bigwedge_{i \in I} \varepsilon\left(f_{i}\right) \\
\alpha \text { is opening } & \Longleftrightarrow \alpha \text { is increasing, idempotent, and anti-extensive } \\
\beta \text { is closing } & \Longleftrightarrow \beta \text { is increasing, idempotent, and extensive }
\end{aligned}
$$

where $I$ is an arbitrary index set.

The above definitions allow broad classes of signal operators to be grouped as lattice dilations, erosions, openings, or closing and their common properties to be studied under the unifying lattice framework. Thus, the translation-invariant morphological dilations, erosions, openings, and closings we saw before are simply special cases of their lattice counterparts. Next, we see some examples and applications of the above general definitions.

EXAMPLE 74.5: Dilation and Translation-Invariant (DTI) Systems

Consider a signal operator that is shift-invariant and obeys a supremum-of-sums superposition:

$$
\mathcal{D}\left[\bigvee_{i} c_{i}+f_{i}(x)\right]=\bigvee_{i} c_{i}+\mathcal{D}\left[f_{i}(x)\right]
$$


Then $\mathcal{D}$ is both a lattice dilation and translation-invariant. We call it a DTI system in analogy to linear time-invariant (LTI) systems that are shift-invariant and obey a linear (sum-of-products) superposition. As an LTI system corresponds in the time-domain to a linear convolution with its impulse response, a DTI system can be represented as a supremal convolution with its upper 'impulse response' $g_{\vee}(x)$ defined as its output when the input is the upper zero impulse $l(x)$, defined in Table 74.4. Specifically,

$$
\mathcal{D} \text { is DTI } \Longleftrightarrow \mathcal{D}(f)=f \oplus g_{\vee}, \quad g_{\vee} \equiv \mathcal{D}(l)
$$

A similar class is the erosion and translation-invariant (ETI) systems $\varepsilon$ which are shift-invariant and obey an infimum-of-sums superposition as in (74.30) but with $\vee$ replaced by $\wedge$. Such systems are equivalent to infimal convolutions with their lower impulse response $g_{\wedge}=\varepsilon(-l)$, defined as the system's output due to the lower impulse $-l(x)$. Thus, DTI and ETI systems are uniquely determined in the time/spatial domain by their impulse responses, which also control their causality and stability [37].

TABLE 74.4 Examples of Upper Slope Transform

\begin{tabular}{|c|c|}
\hline Signal: $f(x)$ & Transform: $F_{\bigvee}(a)$ \\
\hline$\imath\left(x-x_{0}\right) \equiv 0$ if $x=x_{0}$, and $-\infty$ else & $-a x_{0}$ \\
\hline$a_{0} x$ & $-l\left(a-a_{0}\right)$ \\
\hline$\lambda(x) \equiv 0$ if $x \geq 0$, and $-\infty$ else & $-\lambda(a)$ \\
\hline$a_{0} x+\lambda(x)$ & $-\lambda\left(a-a_{0}\right)$ \\
\hline $\begin{cases}0, & |x| \leq r \\
-\infty, & |x|>r\end{cases}$ & $r|a|$ \\
\hline$-a_{0}|x|, \quad a_{0}>0$ & $\begin{array}{ll}0, & |a| \leq a_{0} \\
+\infty, & |a|>a_{0}\end{array}$ \\
\hline$\sqrt{1-x^{2}},|x| \leq 1$ & $\sqrt{1+a^{2}}$ \\
\hline $\begin{array}{c}-\left(|x|^{p}\right) / p, p>1 \\
\exp (x)\end{array}$ & $\begin{array}{c}\left(|a|^{q}\right) / q, \quad 1 / p+1 / q=1 \\
a(1-\log a)\end{array}$ \\
\hline
\end{tabular}

EXAMPLE 74.6: Shift-Varying Dilation

Let $\delta_{B}(f)=f \oplus B$ be the shift-invariant flat dilation of (74.8). In applying it to nonstationary signals, the need may arise to vary the moving window $B$ by actually having a family of windows $B(x)$, possibly varying at each location $x$. This creates the new operator

$$
\delta_{B}(f)(x)=\bigvee_{y \in B(x)} f(x-y)
$$

which is still a lattice dilation, i.e., it distributes over suprema, but it is shift-varying.

\section{EXAMPLE 74.7: Adjunctions}

An operator pair $(\varepsilon, \delta)$ is called an adjunction if $\delta(f) \leq g \leq \Longleftrightarrow f \leq \varepsilon(g)$ for all $f, g \in \mathcal{L}$. Given a dilation $\delta$, there is a unique erosion $\varepsilon$ such that $(\varepsilon, \delta)$ is adjunction, and vice versa. Further, if $(\varepsilon, \delta)$ is an adjunction, then $\delta$ is a dilation, $\varepsilon$ is an erosion, $\delta \varepsilon$ is an opening, and $\varepsilon \delta$ is a closing. Thus, from any adjunction we can generate an opening via the composition of its erosion and dilation. If $\varepsilon$ and $\delta$ are the translation-invariant morphological erosion and dilation in (74.11) and (74.10), then $\delta \varepsilon$ coincides with the translation-invariant morphological opening of (74.13). But there are also numerous other possibilities. 
EXAMPLE 74.8: Radial Opening

If a 2D image $f$ contains $1 \mathrm{D}$ objects, e.g., lines, and $B$ is a $2 \mathrm{D}$ convex structuring element, then the opening or closing of $f$ by $B$ will eliminate these $1 \mathrm{D}$ objects. Another problem arises when $f$ contains large-scale objects with sharp corners that need to be preserved; in such cases opening or closing $f$ by a disk $B$ will round these corners. These two problems could be avoided in some cases if we replace the conventional opening with

$$
\alpha(f)=\bigvee_{\theta} f \circ L_{\theta}
$$

where the sets $L_{\theta}$ are rotated versions of a line segment $L$ at various angles $\theta \in[0,2 \pi)$. The operator $\alpha$, called radial opening, is a lattice opening in the sense of (74.28). It has the effect of preserving an object in $f$ if this object is left unchanged after the opening by $L_{\theta}$ in at least one of the possible orientations $\theta$.

\section{EXAMPLE 74.9: Opening by Reconstruction}

Consider a set $X=\bigcup_{i} X_{i}$ as a union of disjoint connected components $X_{i}$ and let $M \subseteq X_{j}$ be a marker in the $j$ th component; i.e., $M$ could be a single point or some feature set in $X$ that lies only in $X_{j}$. Then, define the conditional dilation of $M$ by $B$ within $X$ as

$$
\delta_{B \mid X}(M) \equiv(M \oplus B) \cap X
$$

If $B$ is a disk with a radius smaller than the distance between $X_{j}$ and any of the other components, then by iterating this conditional dilation we can obtain in the limit

$$
M R_{B \mid X}(M)=\lim _{n \rightarrow \infty} \underbrace{\left(\delta_{B \mid X} \cdots\left(\delta_{B \mid X}\left(\delta_{B \mid X}(M)\right)\right)\right.}_{n \text { times }}
$$

the whole component $X_{j}$. The operator $M R$ is a lattice opening, called opening by reconstruction, and its output is called the morphological reconstruction of the component from the marker. An example is shown in Fig. 74.3. It can extract large-scale components of the image from knowledge only of a smaller marker inside them.

\subsection{Slope Transforms}

Fourier transforms are among the most useful linear signal transformations because they enable us to analyze the processing of signals by linear time-invariant (LTI) systems in the frequency domain, which could be more intuitive or easier to implement. Similarly, there exist some nonlinear signal transformations, called slope transforms, which allow the analysis of the dilation and erosion translation-invariant (DTI and ETI) systems in a transform domain, the slope domain. First, we note that the lines $f(x)=a x+b$ are eigenfunctions of any DTI system $\mathcal{D}$ or ETI system $\mathcal{E}$ because

$$
\begin{aligned}
& \mathcal{D}[a x+b]=a x+b+G_{\vee}(a), G_{\vee}(a) \equiv \bigvee_{x} g_{\vee}(x)-a x \\
& \mathcal{E}[a x+b]=a x+b+G_{\wedge}(a), G_{\wedge}(a) \equiv \bigwedge_{x} g_{\wedge}(x)-a x
\end{aligned}
$$

with corresponding eigenvalues $G_{\vee}(a)$ and $G_{\wedge}(a)$, which are called, respectively, the upper and lower slope response of the DTI and ETI system. They measure the amount of shift in the intercept of the input lines with slope $a$ and are conceptually similar to the frequency response of LTI systems. 


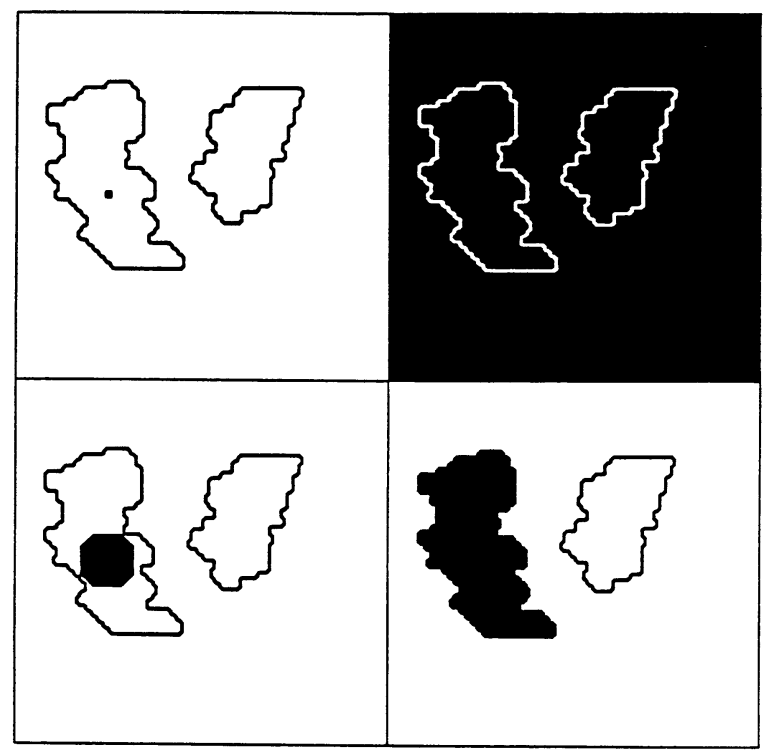

FIGURE 74.3: Let $X$ be the union of the two region boundaries in the top left image, and let $M$ be the single-point marker inside the left region. Top right shows the complement $X^{c}$. If $Y_{0}=M$ and $B$ is a disk-like set whose radius does not exceed the width of the region boundary, iterating the conditional dilation $Y_{i}=\left(Y_{i-1} \oplus B\right) \cap X^{c}$, for $i=1,2,3, \ldots$, yields in the limit (reached at $i=18$ in this case) the interior $Y_{\infty}$ of the left region via morphological reconstruction, shown in bottom right. (Bottom left shows an intermediate result for $i=9$.)

Then, by viewing the slope response as a signal transform with variable the slope $a \in \mathbb{R}$, we define [37] for a 1D signal $f: \mathbb{D} \rightarrow \overline{\mathbb{R}}$ its upper slope transform $F_{\vee}$ and its lower slope transform ${ }^{2}$ $F_{\wedge}$ as the functions

$$
\begin{aligned}
& F_{\vee}(a) \equiv \bigvee_{x \in \mathbb{D}} f(x)-a x \\
& F_{\wedge}(a) \equiv \bigwedge_{x \in \mathbb{D}} f(x)-a x
\end{aligned}
$$

Since $f(x)-a x$ is the intercept of a line with slope $a$ passing from the point $(x, f(x))$ on the signal's graph, for each $a$ the upper (lower) slope transform of $f$ is the maximum (minimum) value of this intercept, which occurs when the above line becomes a tangent. Examples of slope transforms are shown in Fig. 74.4. For differentiable signals, $f$, the maximization or minimization of the intercept $f(x)-a x$ can also be done by finding the stationary point(s) $x^{*}$ such that $d f\left(x^{*}\right) / d x=a$. This extreme value of the intercept is the Legendre transform of $f$ :

$$
F_{L}(a) \equiv f\left((d f / d x)^{-1}(a)\right)-a\left[(d f / d x)^{-1}(a)\right]
$$

It is extensively used in mathematical physics. If the signal $f(x)$ is concave or convex and has an invertible derivative, its Legendre transform is single-valued and equal (over the slope regions it is

\footnotetext{
${ }^{2}$ In convex analysis [50], to a convex function $h$ there uniquely corresponds its Fenchel conjugate $h^{*}(a)=\bigvee_{x} a x-h(x)$, which is the negative of the lower slope transform of $h$.
} 
defined) to the upper or lower transform; e.g., see the last three examples in Table 74.4. If $f$ is neither convex nor concave or if it does not have an invertible derivative, its Legendre transform becomes a set $F_{L}(a)=\left\{f\left(x^{*}\right)-a x^{*}: d f\left(x^{*}\right) / d x=a\right\}$ of real numbers for each $a$. This multivalued Legendre transform, defined and studied in [19] as a 'slope transform', has properties similar to those of the upper/lower slope transform, but there are also some important differences [37].

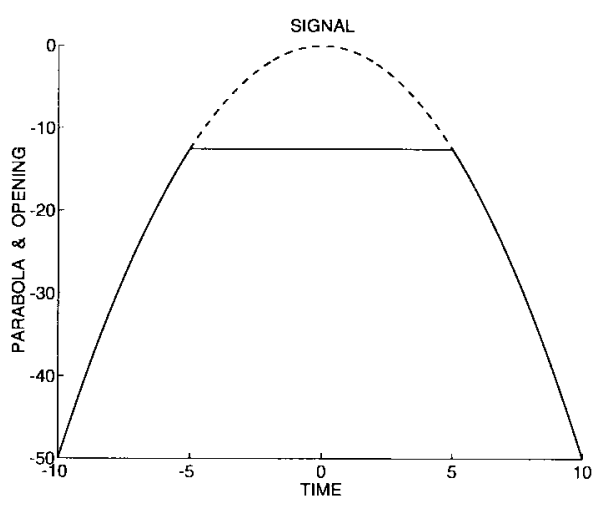

(a)

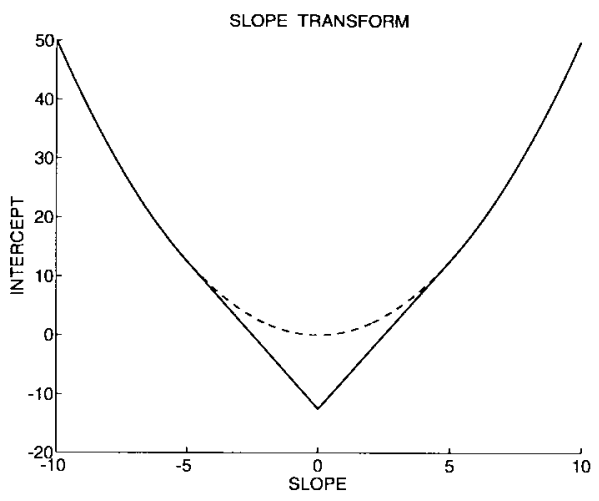

(b)

FIGURE 74.4: (a) Original parabola signal $f(x)=-x^{2} / 2$ (in dashed line) and its morphological opening (in solid line) by a flat structuring element $[-5,5]$. (b) Upper slope transform $F_{\vee}(a)$ of the parabola (in dashed line) and of its opening (in solid line).

The upper and lower slope transform have a limitation in that they do not admit an inverse for arbitrary signals. The closest to an 'inverse' upper slope transform is

$$
\hat{f}(x) \equiv \bigwedge_{a \in \mathbb{R}} F_{\vee}(a)+a x
$$

which is equal to $f$ only if $f$ is concave; otherwise, $\hat{f}$ covers $f$ from above by being its smallest concave upper envelope. Similarly, the supremum over $a$ of all lines $F_{\wedge}(a)+a x$ creates the greatest convex lower envelope $\check{f}(x)$ of $f$, which plays the role of an "inverse" lower slope transform and is equal to $f$ only if $f$ is convex. Thus, for arbitrary signals we have $\check{f} \leq f \leq \hat{f}$.

Tables 74.4 and 74.5 list several examples and properties of the upper slope transform. The most striking is that (dilation) supremal convolution in the time/space domain corresponds to addition in the slope domain. Note the analogy with LTI systems where linearly convolving two signals corresponds to multiplying their Fourier transforms. Very similar properties also hold for the lower slope transform, the only differences being the interchange of suprema with infima, concave with convex, and the supremal $\oplus$ with the infimal convolution $\square$.

The upper/lower slope transforms for discrete-domain and/or multi-dimensional signals are defined as in the 1D continuous case by replacing the real variable $x$ with an integer and/or multidimensional variable, and their properties are very similar or identical to the ones for signals defined on $\mathbb{R}$. See $[37,38]$ for details.

One of the most useful applications of LTI systems and Fourier transform is the design of frequencyselective filters. Similarly, it is also possible to design morphological systems that have a slope selectivity. Imagine a DTI system that rejects all line components with slopes in the band $\left[-a_{0}, a_{0}\right]$ 
TABLE 74.5 Properties of Upper Slope

\begin{tabular}{|c|c|}
\hline Signal: $f(x)$ & Transform: $F_{\vee}(a)$ \\
\hline$\vee_{i} c_{i}+f_{i}(x)$ & $\vee_{i} c_{i}+F_{i}(a)$ \\
\hline $\begin{array}{c}f\left(x-x_{0}\right) \\
f(x)+a_{0} x\end{array}$ & $\begin{array}{c}F(a)-a x_{0} \\
F\left(a-a_{0}\right)\end{array}$ \\
\hline$f(r x)$ & $F(a / r)$ \\
\hline$f(x) \oplus g(x)$ & $F(a)+G(a)$ \\
\hline$\vee_{y} f(x)+g(x+y)$ & $F(-a)+G(a)$ \\
\hline$f(x) \leq g(x) \forall x$ & $F(a) \leq G(a) \forall a$ \\
\hline$g(x)= \begin{cases}f(x), & |x| \leq r \\
-\infty, & |x|>r\end{cases}$ & $G(a)=F(a) \square r|a|$ \\
\hline
\end{tabular}

and passes all the rest unchanged. Then its slope response would be

$$
G(a)=0 \text { if }|a| \leq a_{0} \text {, and }+\infty \text { else . }
$$

This is an ideal-cutoff slope bandpass filter. In the time domain it acts as a supremal convolution with its impulse response

$$
g(x)=-a_{0}|x|
$$

However, $f \oplus g$ is a non-causal infinite-extent dilation, and hence not realizable. Instead, we could implement it as a cascade of a causal dilation by the half-line $g_{1}(x)=-a_{0} x+\lambda(x)$ followed by an anti-causal dilation by another half-line $g_{2}(x)=a_{0} x+\lambda(-x)$, where $\lambda(x)$ is the zero step defined in Table 74.4. This works because $g=g_{1} \oplus g_{2}$. For a discrete-time signal $f[x]$, this slope-bandpass filtering could be implemented via the recursive max-sum difference equation $f_{1}[x]=\max \left(f_{1}[x]-\right.$ $\left.a_{0}, f[x]\right)$ run forward in time, followed by another difference equation $f_{2}[x]=\max \left(f_{2}[x+1]+\right.$ $\left.a_{0}, f_{1}[x]\right)$ run backward in time. The final result would be $f_{2}=f \oplus g$. Such slope filters are useful for envelope estimation [37].

\subsection{Multiscale Morphological Image Analysis}

Multiscale signal analysis has recently emerged as a useful framework for many computer vision and signal processing tasks. Examples include: (1) detecting geometrical features or other events at large scales and then refining their location or value at smaller scales, (2) video and audio data compression using multiband frequency analysis, and (3) measurements and modeling of fractal signals. Most of the work in this area has obtained multiscale signal versions via linear multiscale smoothing, i.e., convolutions with a Gaussian with a variance proportional to scale [15, 53, 72]. There is, however, a variety of nonlinear smoothing filters, including the morphological openings and closings $[35,42,58]$ that can provide a multiscale image ensemble and have the advantage over the linear Gaussian smoothers that they do not blur or shift edges, as shown in Fig. 74.5. There we see that the gray-level close-openings by reconstruction are especially useful because they can extract the exact outline of a certain object by locking on it while smoothing out all its surroundings; these nonlinear smoothers have been applied extensively in multiscale image segmentation [56]. The use of morphological operators for multiscale signal analysis is not limited to operations of a smoothing type; e.g., in fractal image analysis, erosion and dilation can provide multiscale distributions of the shrink-expand type from which the fractal dimension can be computed [36].

Overall, many applications of morphological signal processing such as nonlinear smoothing, geometrical feature extraction, skeletonization, size distributions, and segmentation, inherently require or can benefit from performing morphological operations at multiples scales. The required building blocks for a morphological scale-space are the multiscale dilations and erosions. Consider a planar 


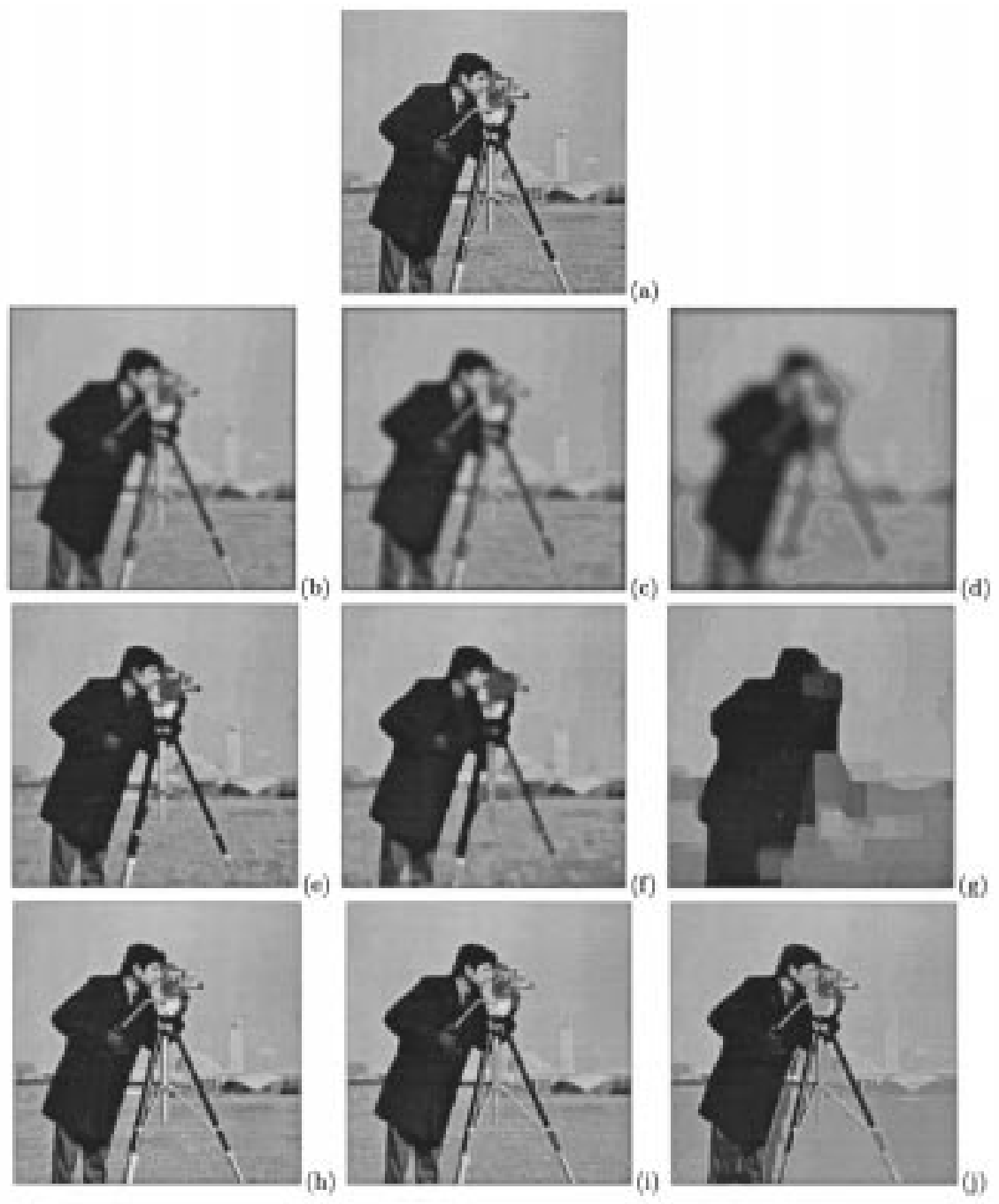

FIGURE 74.5: (a) Original image and its multiscale smoothings via: (b,c,d) Gaussian convolution at scales $2,4,16$; (e,f,g) close-opening by a square at scales $2,4,16$; (h,i,j) close-opening by reconstruction at scales $2,4,16$. 
compact convex set $B=\left\{(x, y):\|(x, y)\|_{p} \leq 1\right\}$ that is the unit ball generated by the $L_{p}$ norm, $p=1,2, \ldots, \infty$. Then the simplest multiscale dilation and erosion of a signal $f(x, y)$ at scales $t>0$ are the multiscale flat sup/inf convolutions by $t B=\{t z: z \in B\}$

$$
\begin{aligned}
& \delta(x, y, t) \equiv(f \oplus t B)(x, y) \\
& \varepsilon(x, y, t) \equiv(f \ominus t B)(x, y)
\end{aligned}
$$

which apply both to gray-level and binary images.

\subsubsection{Binary Multiscale Morphology via Distance Transforms}

Viewing the boundaries of multiscale erosions/dilations of a binary image by disks as wavefronts propagating from the original image boundary at uniform unit normal velocity and assigning to each pixel the time $t$ of wavefront arrival creates a distance function, called the distance transform [10]. This transform is a compact way to represent their multiscale dilations and erosions by disks and other polygonial structuring elements whose shape depends on the norm $\|\cdot\|_{p}$ used to measure distances. Formally, the distance transform of the foreground set $F$ of a binary image is defined as

$$
D_{p}(F)(x, y) \equiv \bigwedge_{(v, u) \in F^{c}}\left\{\|(x-v, y-u)\|_{p}\right\}
$$

Thresholding the distance transform at various levels $t>0$ yields the erosions of the foreground $F$ (or the dilation of the background $F^{c}$ ) by the norm-induced ball $B$ at scale $t$ :

$$
F \ominus t B=\Theta_{t}\left[D_{p}(F)\right]
$$

Another view of the distance transform results from seeing it as the infimal convolution of the $(0,+\infty)$ indicator function of $F^{c}$,

$$
I_{F^{c}}(x) \equiv 0 \text { if } x \in F^{c}, \text { and }+\infty \text { else, }
$$

with the norm-induced conical structuring function:

$$
D_{p}(F)(x)=I_{F^{c}}(x) \square\|x\|_{p}
$$

Recognizing $g_{\wedge}(x)=\|x\|_{p}$ as the lower impulse response of an ETI system with slope response

$$
G_{\wedge}(a)=0 \text { if }\|a\|_{q} \leq 1, \text { and }-\infty \text { else },
$$

where $1 / p+1 / q=1$, leads to seeing the distance transform as the output of an ideal-cutoff slopeselective filter that rejects all input planes whose slope vector falls outside the unit ball with respect to the $\|\cdot\|_{q}$ norm, and passes all the rest unchanged.

To obtain isotropic distance propagation, the Euclidean distance transform is desirable because it gives multiscale morphology with the disk as the structuring element. However, since this has a significant computational complexity, various techniques are used to obtain approximations to the Euclidean distance transform of discrete images at a lower complexity. A general such approach is the use of discrete distances [54] and their generalization via chamfer metrics [11]. Given a discrete binary image $f[i, j] \in\{0,+\infty\}$ with 0 marking background/source pixels and $+\infty$ marking foreground/object pixels, its global chamfer distance transform is obtained by propagating local distances within a small neighborhood mask. An efficient method to implement it is a two-pass sequential algorithm [11,54] where for a $3 \times 3$ neighborhood the min-sum difference equation

$$
\begin{aligned}
& u_{n}[i, j]=\min \left(u_{n-1}[i, j], u_{n}[i-1, j]+a, u_{n}[i, j-1]+a,\right. \\
& \left.u_{n}[i-1, j-1]+b, u_{n}[i+1, j-1]+b\right)
\end{aligned}
$$


is run recursively over the image domain: first $(n=1)$, in a forward scan starting from $u_{0}=f$ to obtain $u_{1}$, and second $(n=2)$ in a backward scan on $u_{1}$ using a reflected mask to obtain $u_{2}$, which is the final distance transform. The coefficients $a$ and $b$ are the local distances within the neighborhood mask. The unit ball associated with chamfer metrics is a polygon whose approximation of the disk improves by increasing the size of the mask and optimizing the local distances so as to minimize the error in approximating the true Euclidean distances. In practice, integer-valued local distances are used for faster implementation of the distance transform. If $(a, b)$ is $(1,1)$ or $(1, \infty)$, the chamfer ball becomes a square or rhombus, respectively, and the chamfer distance transform gives poor approximations to multiscale morphology with disks. The commonly used ( $a=3, b=4)$ chamfer metric gives a maximum absolute error of about $6 \%$, but even better approximations can be found by optimizing $a, b$.

\subsubsection{Multiresolution Morphology}

In certain multiscale image analysis tasks, the need also arises to subsample the multiscale image versions and thus create a multiresolution pyramid [15, 53]. Such concepts are very similar to the ones encountered in classical signal decimation. Most research in image pyramids has been based on linear smoothers. However, since morphological filters preserve essential shape features, they may be superior in many applications. A theory of morphological decimation and interpolation has been developed in [25] to address these issues which also provides algorithms on reconstructing a signal after morphological smoothing and decimation with quantifiable error. For example, consider a binary discrete image represented by a set $X$ that is smoothed first to $Y=X \circ B$ via opening and then down-sampled to $Y \cap S$ by intersecting it with a periodic sampling set $S$ (satisfying certain conditions). Then the Hausdorff distance between the smoothed signal $Y$ and the interpolation (via dilation) $(Y \cap S) \oplus B$ of its down-sampled version does not exceed the radius of $B$. These ideas also extend to multilevel signals.

\subsection{Differential Equations for Continuous-Scale Morphology}

Thus far, most of the multiscale image filtering implementations have been discrete. However, due to the current interest in analog VLSI and neural networks, there is renewed interest in analog computation. Thus, continuous models have been proposed for several computer vision tasks based on partial differential equations (PDEs). In multiscale linear analysis [72] a continuous (in scale $t$ and spatial argument $x, y$ ) multiscale signal ensemble

$$
\gamma(x, y, t)=f(x, y) * G_{t}(x, y), G_{t}(x, y)=\frac{\exp \left[-\left(x^{2}+y^{2}\right) / 4 t\right]}{\sqrt{4 \pi t}}
$$

is created by linearly convolving an original signal $f$ with a multiscale Gaussian function $G_{t}$ whose variance $(2 t)$ is proportional to the scale parameter $t$. The Gaussian multiscale function $\gamma$ can be generated [28] from the linear diffusion equation

$$
\frac{\partial \gamma}{\partial t}=\frac{\partial^{2} \gamma}{\partial x^{2}}+\frac{\partial^{2} \gamma}{\partial y^{2}}
$$

starting from the initial condition $\gamma(x, y, 0)=f(x, y)$.

Motivated by the limitations or inability of linear systems to successfully model several image processing problems, several nonlinear PDE-based approaches have been developed. Among them, some PDEs have been recently developed to model multiscale morphological operators as dynamical systems evolving in scale-space $[1,14,66]$. 
Consider the multiscale morphological flat dilation and erosion of a 2D image signal $f(x, y)$ by the unit-radius disk at scales $t \geq 0$ as the space-scale functions $\delta(x, y, t)$ and $\varepsilon(x, y, t)$ of $(74.43)$ and (74.44). Then [14] the PDE generating these multiscale flat dilations is

$$
\frac{\partial \delta}{\partial t}=\|\nabla \delta\|=\sqrt{\left(\frac{\partial \delta}{\partial x}\right)^{2}+\left(\frac{\partial \delta}{\partial y}\right)^{2}}
$$

and for the erosions is $\partial \varepsilon / \partial t=-\|\nabla \varepsilon\|$. These morphological PDEs directly apply to binary images because flat dilations/erosions commute with thresholding and hence, when the gray-level image is dilated/eroded, each one of its thresholded versions representing a binary image is simultaneously dilated/eroded by the same element and at the same scale.

In equivalent formulations $[10,57,66]$, the boundary of the original binary image is considered as a closed curve and this curve is expanded perpendicularly at constant unit speed. The dilation of the original image with a disk of radius $t$ is the expanded curve at time $t$. This propagation of the image boundary is a special case of more general curvature-dependent propagation schemes for curve evolution studied in [47]. This general curve evolution methodology was applied in [57] to obtain multiscale morphological dilations/erosions of binary images, using an algorithm [47] where the original curve is first embedded in the surface of a $2 \mathrm{D}$ continuous function $\Phi_{0}(x, y)$ as its zero level set and then the evolving $2 \mathrm{D}$ curve is obtained as the zero level set of a $2 \mathrm{D}$ function $\Phi(x, y, t)$ that evolves from the initial condition $\Phi(x, y, 0)=\Phi_{0}(x, y)$ according to the PDE $\partial \Phi / \partial t=\|\nabla \Phi\|$. This function evolution PDE makes zero level sets expand at unit normal speed and is identical to the PDE (74.53) for flat dilation by disk. The main steps in its numerical implementations [47] are:

$$
\begin{aligned}
\Phi_{i, j}^{n} & =\text { estimate of } \Phi\left(i \Delta x, j \Delta_{y}, n \Delta t\right) \text { on a grid } \\
D_{x}^{+} & =\left(\Phi_{i+1, j}^{n}-\Phi_{i, j}^{n}\right) / \Delta x, D_{x}^{-}=\left(\Phi_{i, j}^{n}-\Phi_{i-1, j}^{n}\right) / \Delta x \\
D_{y}^{+} & =\left(\Phi_{i, j+1}^{n}-\Phi_{i, j}^{n}\right) / \Delta y, D_{y}^{-}=\left(\Phi_{i, j}^{n}-\Phi_{i, j-1}^{n}\right) / \Delta y \\
G^{2} & =\min ^{2}\left(0, D_{x}^{-}\right)+\max ^{2}\left(0, D_{x}^{+}\right)+\min ^{2}\left(0, D_{y}^{-}\right)+\max ^{2}\left(0, D_{y}^{+}\right) \\
\Phi_{i, j}^{n} & =\Phi_{i, j}^{n-1}+G \Delta t, n=1,2, \ldots,(R / \Delta t)
\end{aligned}
$$

where $R$ is the maximum scale (radius) of interest, $\Delta x, \Delta y$ are the spatial grid spacings, and $\Delta t$ is the time (scale) step.

Continuous multiscale morphology using the above curve evolution algorithm for numerically implementing the dilation PDE yields better approximations to disks and avoids the abrupt shape discretization inherent in modeling digital multiscale using discrete polygons [16, 57]. Comparing it to discrete multiscale morphology using chamfer distance transforms, we note that for binary images: (1) the chamfer distance transform is easier to implement and yields similar errors for small scale dilations/erosions; (2) implementing the distance transform via curve evolution is more complex, but at medium and large scales gives a better and very close approximation to Euclidean geometry, i.e., to morphological operations with the disk structuring element. See Fig. 74.6.

\subsection{Applications to Image Processing and Vision}

There are numerous applications of morphological image operators to image processing and computer vision. Examples of broad application areas include biomedical image processing, automated visual inspection, character and document image processing, remote sensing, nonlinear filtering, multiscale image analysis, feature extraction, motion analysis, segmentation, and shape recognition. Next we shall review a few of these applications to specific problems of image processing and low/mid-level vision. 


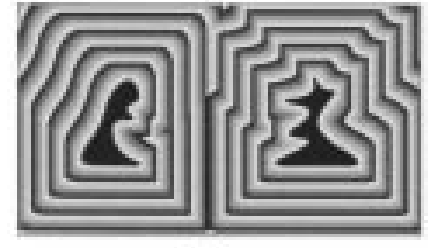

(a)

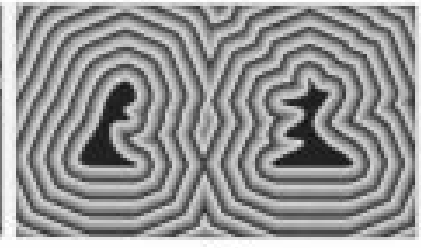

(b)

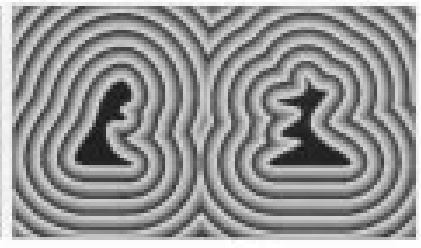

(c)

FIGURE 74.6: Distance transforms of a binary image, shown as intensity images modulo 20, obtained using: (a) Metric $\|\cdot\|_{\infty}$ (chamfer metric with local distances $(1,1)$ ), (b) chamfer metric with $3 \times 3$ neighborhood and local distances $(24,34) / 25$, and (c) curve evolution.

\subsubsection{Noise Suppression}

Rank filters and especially medians have been applied mainly to suppress impulse noise or noise whose probability density has heavier tails than the Gaussian for enhancement of image and other signals $[2,12,27,64,65]$, since they can remove this type of noise without blurring edges, as would be the case for linear filtering. The rank filters have also been used for envelope detection. In their behavior as nonlinear smoothers, as shown in Fig. 74.7, the medians act similarly to an 'open-closing' $(f \circ B) \bullet B$ by a convex set $B$ of diameter about half the diameter of the median window. The openclosing has the advantages over the median that it requires less computation and decomposes the noise suppression task into two independent steps, i.e., suppressing positive spikes via the opening and negative spikes via the closing. Further, cascading open-closings $\beta_{t} \alpha_{t}$ at multiple scales $t=1, \ldots, r$, where $\alpha_{t}(f)=f \circ t B$ and $\beta_{t}(f)=f \bullet t B$, generates a class of efficient nonlinear smoothing filters $\beta_{r} \alpha_{r} \ldots \beta_{2} \alpha_{2} \beta_{1} \alpha_{1}$, called alternating sequential filters, which smooth progressively from the smallest scale possible up to a maximum scale $r$ and have a broad range of applications [59, 60, 62].

\subsubsection{Feature Extraction}

Residuals between a signal and some morphologically transformed versions of it can extract line- or blob-type features or enhance their contrast. An example is the difference between the flat dilation and erosion of an image $f$ by a symmetric disk-like set $B$ whose diameter, $\operatorname{diam}(B)$, is very small;

$$
\text { edge }(f)=\frac{(f \oplus B)-(f \ominus B)}{\operatorname{diam}(B)}
$$

If $f$ is binary, edge $(f)$ extracts its boundary. If $f$ is gray-level, the above residual enhances its edges $[7,58]$ by yielding an approximation to $\|\nabla f\|$, which is obtained in the limit of (74.54) as $\operatorname{diam}(B) \rightarrow 0$. See Fig. 74.8. This morphological edge operator can be made more robust for edge detection by first smoothing the input image signal and compares favorably with other gradient approaches based on linear filtering.

Another example involves subtracting the opening of a signal $f$ by a compact convex set $B$ from the input signal yields an output consisting of the signal peaks whose support cannot contain $B$. This is the top-hat transformation [43, 58]

$$
\text { peak }(f)=f-(f \circ B)
$$

and can detect bright blobs, i.e., regions with significantly brighter intensities relative to the surroundings. Similarly, to detect dark blobs, modeled as intensity valleys, we can use the closing residual operator $f \mapsto(f \bullet B)-f$. See Fig. 74.8. The morphological peak/valley extractors, in addition to their being simple and efficient, have some advantages over curvature-based approaches. 


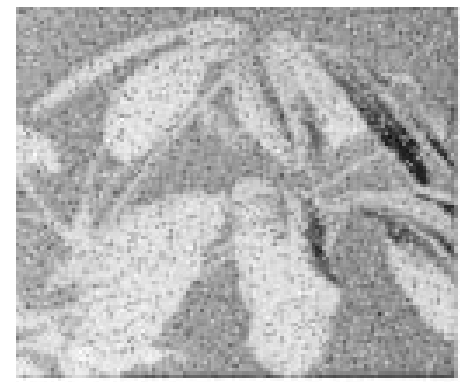

(a)

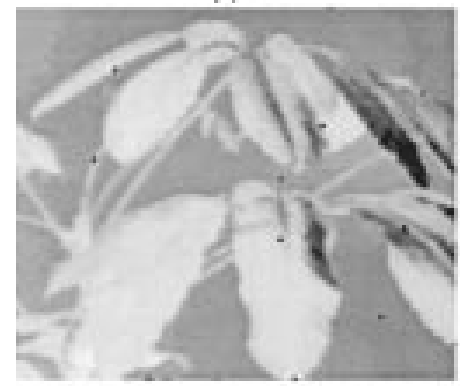

(c)

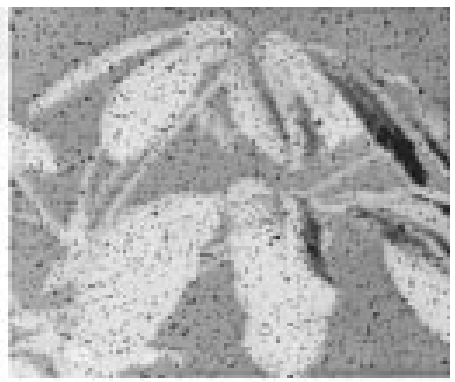

(b)

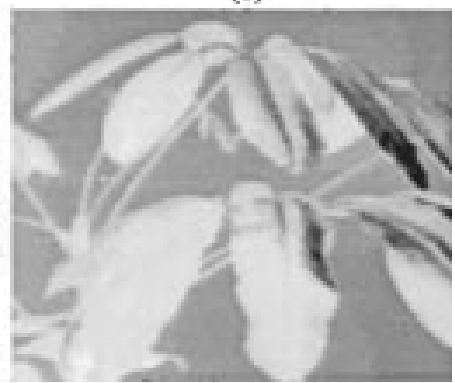

(d)

FIGURE 74.7: (a) Noisy image $f$, corrupted with salt-and-pepper noise of probability $10 \%$. (b) Opening $f \circ B$ of $f$ by a $2 \times 2$-pixel square $B$. (c) Open-closing $(f \circ B) \bullet B$. (d) Median of $f$ by a $3 \times 3$-pixel square window.

\subsubsection{Shape Representation via Skeleton Transforms}

There are applications in image processing and vision where a binary shape needs to be summarized down to its thin medial axis and then reconstructed exactly from this axial information. This process, known as medial axis (or skeleton) transform has been studied extensively for shape representation and description [10,54]. Among many approaches, it can also be obtained via multiscale morphological operators, which offer as a by-product a multiscale representation of the original shape via its skeleton components [39, 58]. Let $X \subseteq \mathbb{Z}^{2}$ represent the foreground of a finite discrete binary image and let $B \subseteq \mathbb{Z}^{2}$ be a convex disk-like set at scale 1 and $B^{\oplus n}$ be its multiscale version at scale $n=1,2, \ldots$ The $n$th skeleton component of $X$ is the set

$$
S_{n}=\left(X \ominus B^{\oplus n}\right) \backslash\left[\left(X \ominus B^{\oplus n}\right) \circ B\right], n=0,1, \ldots, N,
$$

where $\backslash$ denotes the difference, $n$ is a discrete scale parameter, and $N=\max \left\{n: X \ominus B^{\oplus n} \neq \emptyset\right\}$ is the maximum scale. The $S_{n}$ are disjoint subsets of $X$, whose union is the morphological skeleton of $X$.

The morphological skeleton transform of $X$ is the finite sequence $\left(S_{0}, S_{1}, \ldots, S_{N}\right)$. The union of all the $S_{n}$ s dilated by a $n$-scale disk reconstructs exactly the original shape; omitting the first $k$ components leads to a smooth partial reconstruction, the opening of $X$ at scale $k$ :

$$
X \circ B^{\oplus k}=\bigcup_{k \leq n \leq N} S_{n} \oplus B^{\oplus n}, 0 \leq k \leq N .
$$

Thus, we can view the $S_{n}$ as 'shape components', where the small-scale components are associated with the lack of smoothness of the boundary of $X$, whereas skeleton components of large scale indices $n$ 


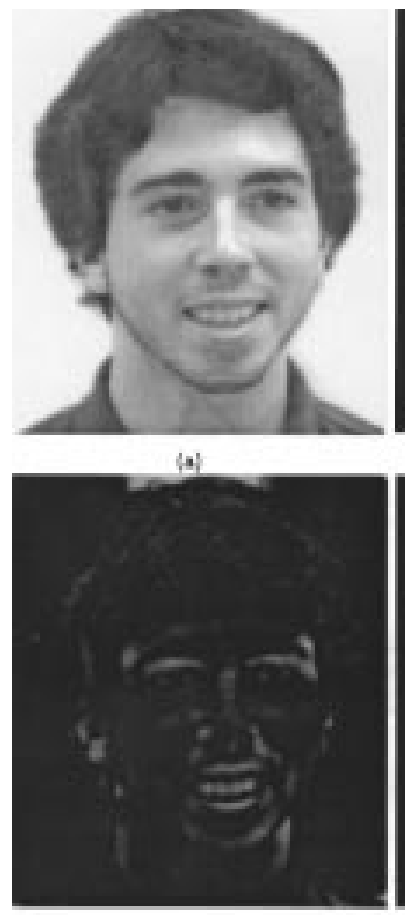

$\{c\}$

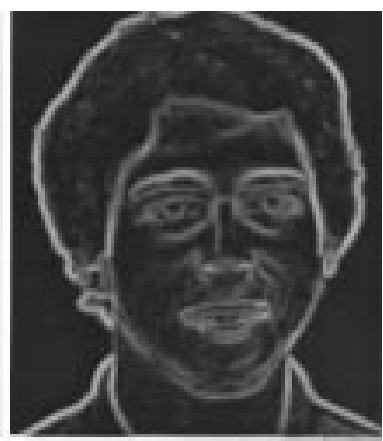

[b]

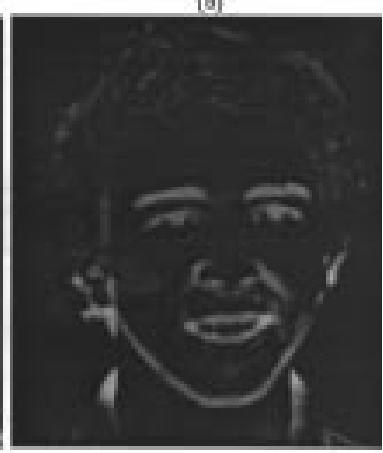

(d)

FIGURE 74.8: (a) Image $f$. (b) Edge enhancement: dilation-erosion residual $f \oplus B-f \ominus B$, where $B$ is a 21-pixel octagon. (c) Peak detection: opening residual $f-f \circ B^{\oplus 3}$. (d) Valley detection: closing residual $f \bullet B^{\oplus 3}-f$.

are related to the bulky interior parts of $X$ that are shaped similarly to $B^{\oplus n}$. Figure 74.9 shows a detailed description of the skeletal decomposition and reconstruction of an image.

Several generalizations or modifications of the morphological skeletonization include: using structuring elements different than disks that might result in fewer skeletal points, or removing redundant points from the skeleton [29, 33, 39]; using different structuring elements for each skeletonization step [23, 33]; using lattice generalizations of the erosions and openings involved in skeletonization [30]; image representation based on skeleton-like multiscale residuals [23]; and shape decomposition based on residuals between image parts and maximal openings [48]. In addition to its general use for shape analysis, a major application of skeletonization has been binary image coding [13, 30, 39].

\subsubsection{Shape Thinning}

The skeleton is not necessarily connected; for connected skeletons see [3]. Another approach for summarizing a binary shape down to a thin medial axis that is connected but does not necessarily guarantee reconstruction is via thinning. Morphological thinning is defined [58] as the difference between the original set $X$ (representing the foreground of a binary image) and a set of feature locations extracted via hit-miss transformations by pairs of foreground-background probing sets 


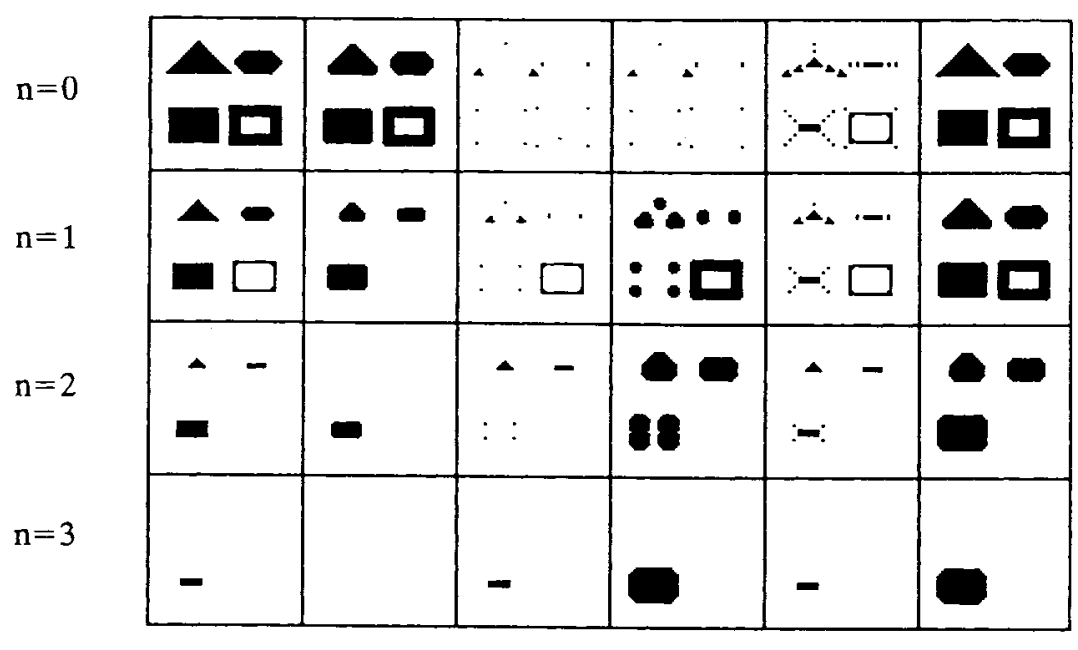

$\begin{array}{lllll}\text { (a) } & \text { (b) } & \text { (c) } & \text { (d) } & \text { (e) }\end{array}$

FIGURE 74.9: Morphological skeletonization of a binary image $X$ (top left image) with respect to a $3 \times 3$-pixel square structuring element $B$. (a) Erosions $X \ominus B^{\oplus n}, n=0,1,2,3$. (b) Openings of erosions $\left(X \ominus B^{\oplus n}\right) \circ B$. (c) Skeleton subsets $S_{n}$. (d) Dilated skeleton subsets $S_{n} \oplus B^{\oplus n}$. (e) Partial unions of skeleton subsets $\cup_{N \geq k \geq n} S_{k}$. (f) Partial unions of dilated skeleton subsets $\cup_{N \geq k \geq n} S_{k} \oplus B^{\oplus k}$.

$\left(A_{i}, B_{i}\right)$ designed to detect features that thicken the shape's axis:

$$
X \bigcirc\left\{\left(A_{i}, B_{i}\right)\right\}_{i=1}^{n} \equiv X \backslash \bigcup_{i=1}^{n} X \otimes\left(A_{i}, B_{i}\right)
$$

Usually each hit-miss by a pair $\left(A_{i}, B_{i}\right)$ detects a feature at some orientation, and then the difference from the original peels off this feature from $X$. Since this feature might occur at several orientations, the above thinning operator is applied iteratively by rotating its set of probing elements until there is no further change in the image. Thinning has been applied extensively to character images. Examples are shown in Fig. 74.10, where each thinning iteration used $n=3$ template pairs $\left(A_{i}, B_{i}\right)$ for the hit-miss transformations of (74.58) designed in [8].

\subsubsection{Size Distributions}

Multiscale openings $X \mapsto X \circ r B$ and closings $X \mapsto X \bullet r B$ of compact sets $X$ in $\mathbb{R}^{d}$ by convex compact structuring elements $r B$, parameterized by a scale parameter $r \geq 0$, are called granulometries and can unify all sizing (sieving) operations [42]. Because they satisfy a monotonic ordering

$$
\ldots X \circ s B \subseteq X \circ r B \subseteq \ldots \subseteq X \subseteq \ldots X \bullet r B \subseteq X \bullet s B \subseteq \ldots, r<s
$$

if we measure the volume (or area) of these sets as a function of scale, this function will also satisfy the same ordering and hence create size distributions. Further, taking its derivative leads to a size 


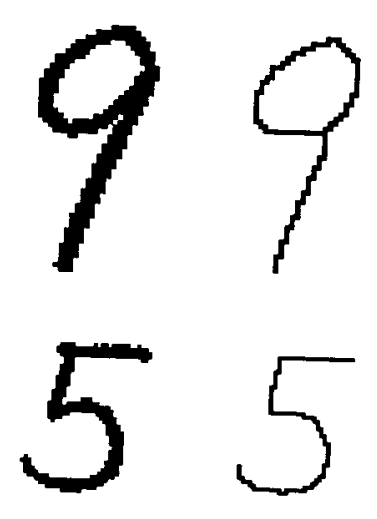

FIGURE 74.10: Left column shows binary images of handwritten characters. Right column shows their thinned version.

density function (or size histogram in the discrete case)

$$
h(r) \equiv \begin{cases}-\frac{d \operatorname{vol}(X \circ r B)}{d r}, & r \geq 0 \\ \frac{d \operatorname{vol}(X \bullet|r| B)}{d|r|}, & r<0\end{cases}
$$

This conveys several types of information useful for shape description and multiscale image analysis. For example, the boundary roughness of $X$ relative to $B$ manifests itself as contributions in the lowersize part of the size histogram. Long capes or bulky protruding parts in $X$ that consist of patterns $s B$ show up as isolated impulses in the histogram around positive $r=s$. Finally, the size density can be defined for 'negative' sizes by using closings instead of openings; in this case impulses at negative sizes indicate the existence of prominent intruding gulfs or holes in $X$.

If $X$ is a random set [42], then probabilistic measures of its size distribution have been used extensively in image analysis applications to petrography and biology [58]. All of the above ideas can be extended to gray-level images [35]. One application of gray-level size distributions is texture classification [17].

\subsubsection{Fractals}

A large variety of natural image objects (e.g., clouds, coastlines, mountains, islands, trees, leaves, etc.) can be modeled with fractals [32]. Fractals are mathematical sets with a very high level of geometrical complexity; formally, their Hausdorff dimension is larger than their topological dimension. An important characteristic of fractals to measure for purposes of shape description or classification is their fractal dimension. Among the various methods [32] to estimate the fractal dimension $D$ of the surface of a set $F \subseteq \mathbb{R}^{3}$, the covering method is based conceptually on Minkowski's idea of finding the area of irregular sets; dilate them with spheres of radius $r$, find the volume $V(r)$ of the dilated set, and set its area equal to $\lim _{r \downarrow 0} A(r)$, where $A(r)=V(r) / 2 r$. Further, the fractal dimension of $F$ can be found by

$$
D=\lim _{r \downarrow 0} \frac{\log \left[V(r) / r^{3}\right]}{\log [(1 / r)]}
$$

The intuitive meaning of $D$ is that $V(r) \approx$ (constant) $\cdot r^{3-D}$ as $r \downarrow 0$, from which $D$ can be estimated by least-squares fitting a straight line to a log-log plot of $V(r)$.

The theory of morphological operators allows us to find more efficient implementations of the above idea when $F$ is the graph of a 2D function $f(x, y)$. Then, instead of multiscale 3D set dilations 
of $F$ by spheres, it is computationally more efficient to perform 2D multiscale signal dilations and erosions of $f$ by disks $r B$ and measure the multiscale volumes by

$$
V(r)=\iint[(f \oplus r B)(x, y)-(f \ominus r B)(x, y)] d x d y
$$

Thus, morphological flat dilations and erosions are used to create a volume-blanket as a layer either covering or being peeled off from the surface of $f$ at various scales. This morphological covering method can also be applied to 1D signals $f(x)$ by replacing volumes with areas and disks $r B$ with horizontal linear segments $[-r, r]$; such a 1D application is shown in Fig. 74.11.

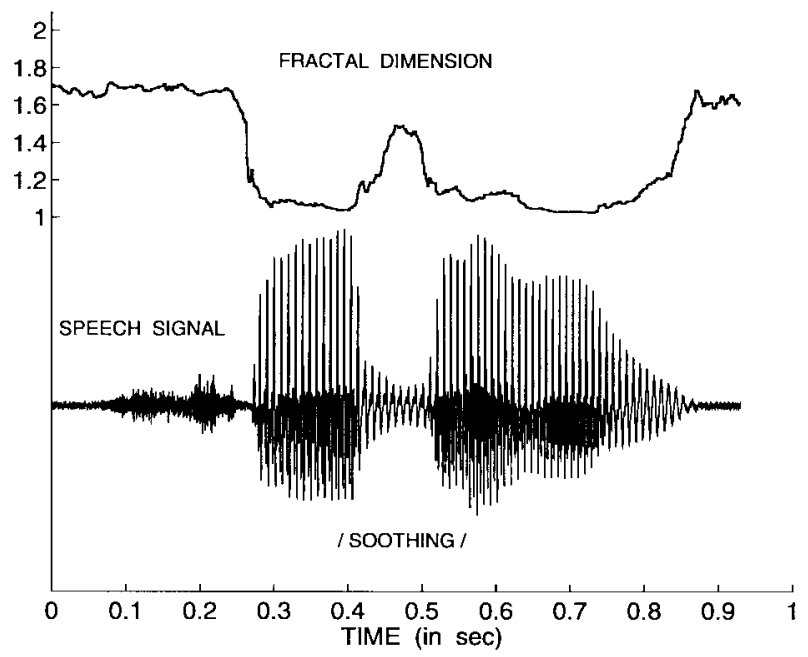

FIGURE 74.11: Speech waveform of the word 'soothing' sampled at $10 \mathrm{kHz}$ and its short-time fractal dimension over 10-ms speech segments, computed every $1 \mathrm{~ms}$ and post-smoothed by a 3-point median filter. The short-time fractal dimension increases with the amount of turbulence existing during production of the corresponding sound, having a small value for vowels, medium for weak voiced fricatives, and high for unvoiced fricatives.

\subsubsection{Image Segmentation}

One of the most powerful and advanced tools of mathematical morphology is the watershed transformation [7] as applied to image segmentation. Let us regard the gray-level image to be segmented as a topographic relief and assume a drop of water falling at a point on it and flowing down along a steep slope path until it is trapped in a local minimum $M$ of the relief. The set of points such that a drop falling on them eventually reaches $M$ is the catchment basin associated with the minimum $M$. The union of the boundaries of the different catchment basins of the image constitute its watershed. Thus, the watershed consists of contours located on crest lines separating adjacent minima.

To ease the segmentation of the original image $f$, the watershed transformation is usually applied to its gradient magnitude $g=\|\nabla f\|$, which has higher contrast. However, direct computation of the watershed of $g$ usually leads to poor results, i.e., oversegmentation of $f$, because, even after smoothing $f$ or $g$, the latter often exhibits far too many minima. One of the best solutions to this 
problem is to use markers for the regions to be extracted. A marker is a small connected component of pixels, a feature, located inside a region. Once the markers have been extracted, the gradient image $g$ is modified via morphological reconstruction so that these markers are imposed as the only minima of the modified function while preserving the highest crest lines of $g$ located between two markers. Then, computing the watershed of the modified $g$ usually provides a good segmentation whose quality depends mainly on the markers and somewhat on $g$ and the initial smoothing of $f$. An example is shown in Fig. 74.12. The power of this approach as well as its difficulty lies in the choice of the markers. Efficient ways to choose markers as well as fast algorithms for the watershed computation are detailed in $[44,69]$. This watershed methodology has already proved to be very useful in various fields of image analysis, ranging from medical imaging to material sciences, remote sensing, and digital elevation models.

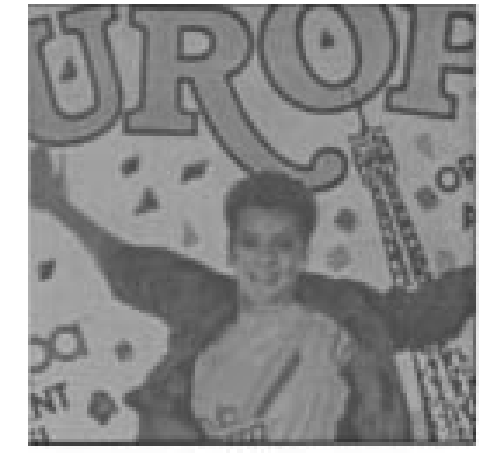

(a)

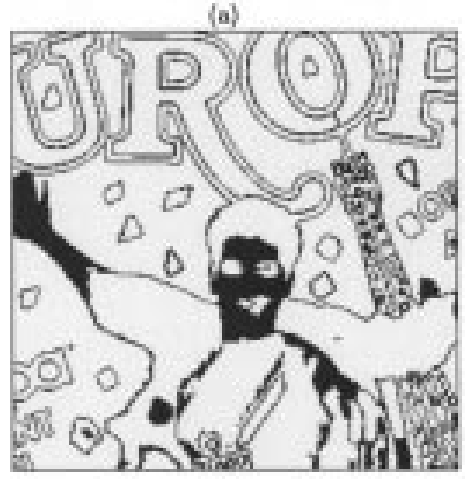

(c)

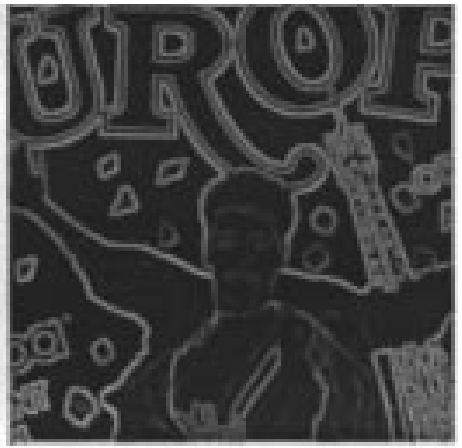

(b)

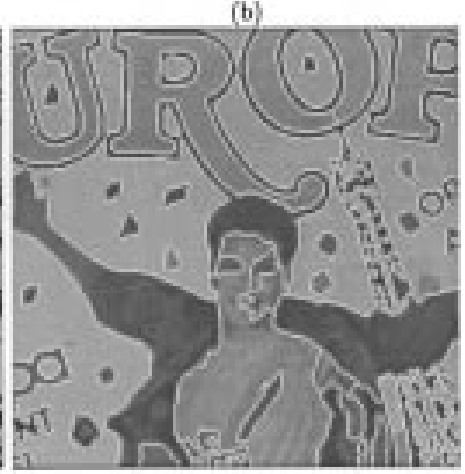

(d)

FIGURE 74.12: (a) Image $f$. (b) Edge enhancement (magnitude of gradient) of $f$. (c) Markers. (d) Watershed.

\subsection{Conclusions}

This chapter has provided a brief introduction of the theory of morphological signal processing and its applications to image analysis and nonlinear filtering. This methodology nowadays offers a large diversity of theoretical and algorithmic ideas that provide useful tools and inspire new directions in the following research areas from the fields of signal processing, image processing and machine vision, and pattern recognition: nonlinear filtering, nonlinear signal and system representation, 
image feature extraction, multiscale analysis and geometry-driven diffusion, image segmentation, region-based image coding, motion analysis, automated visual inspection, and detection/estimation in random sets.

Some attractive aspects of morphological signal operators for efficiently solving problems in the above areas include: (1) suitability for geometry-related signal/image analysis problems; (2) unification power because they can be defined both for numerical signals as well as for more abstract data using their lattice generalizations; (3) simplicity of software or hardware implementations of the basic operators; and (4) existence of efficient algorithms for implementing complex morphological systems [68].

Three current research areas where successful future developments may significantly broaden and improve the applicability of morphological signal processing are: (A) Optimal design of nonlinear systems based on morphological and related signal operators, where, despite their numerous applications, very few ideas exist for their optimal design. (The current three main approaches are: (1) designing binary systems as a finite union of erosions [20,31] or hit-miss operations [5] using the morphological representation theory of [33, 34] or [4]; (2) designing stack filters via threshold decomposition and linear programming [18]; (3) gradient-based optimization of morphological/rank filters either via simulated annealing [71] or via a least-mean-square algorithm and adaptive filtering [55].) (B) The continuous (differential) approach to mathematical morphology via PDEs and exploitation of its exciting relationships to the physics of wave propagation and eikonal optics [38, 57, 67]. (C) Development of morphological systems for image pattern recognition by exploiting the efficiency of morphological operators for shape analysis and their logic-related structure.

\section{Acknowledgment}

This chapter was written while the author's research work was supported by the U.S. National Science Foundation under Grant MIP-94-21677.

\section{References}

[1] Alvarez, L. and Morel, J.M., Formalization and computational aspects of image analysis, Acta Numerica, 1-59, 1994.

[2] Arce, G.R., Gallagher, N.C. and Nodes, T.A., Median filters: Theory for one- and twodimensional filters, in Advances in Computer Vision and Image Processing, Vol.2, Huang, T.S., Ed., JAI Press, Connecticut, 1986.

[3] Arcelli, C., Cordella, L. and Levialdi, S., From local maxima to connected skeletons, IEEE Trans. Pattern Anal. Mach. Intellig., PAMI-3, 134-143, Mar. 1981.

[4] Banon, G.J.F. and Barrera, A.J., Minimal representations for translation-invariant set mappings by mathematical morphology, SIAM J. Appl. Math., 51, 1782-1798, Dec. 1991.

[5] Barrera, A.J., Salas, B.G.P. and Hashimoto, C.R.F., Set operations on closed intervals and their applications to the programming of MMach's, in Mathematical Morphology and its Application to Image and Signal Processing, Maragos, P., Schafer, R.W. and Butt, M.A., Eds., Kluwer Acad. Publishing, 1996.

[6] Bellman, R. and Karush, W., On the maximum transform, J. Math. Anal. Appl., 6, 67-74, 1963.

[7] Beucher, S. and Lantuejoul, C., Use of watersheds in contour detection, Proc. Int'l. Workshop on Image Processing: Real-time Edge \& Motion Detection/Estimation, Rennes, France, 1979.

[8] Bloomberg, D.S., Connectivity-preserving morphological image transformations, in Visual Communications and Image Processing '91, Tzou, K.-H. and Koga, T., Eds., Proc. SPIE, vol. 1606, 1991. 
[9] Bloomberg, D.S., Multiresolution morphological analysis of document images, in Visual Communications and Image Processing'92, Maragos, P., Ed., Proc. SPIE, 1818, 648-662, 1992.

[10] Blum, H., Biological shape and visual science (part I), J. Theor. Biol., 38, 205-287, 1973.

[11] Borgefors, G., Distance transformations in digital images, Comp. Vision, Graphics, Image Process., 34, 344-371, 1986.

[12] Bovik, A.C., Huang, T.S. and Munson, Jr., D.C., A generalization of median filtering using linear combinations of order statistics, IEEE Trans. Acoust. Speech, Signal Process., 31, 1342-1349, Dec. 1983.

[13] Brandt, J.W., Jain, A.K. and Algazi, V.R., Medial axis representation and encoding of scanned documents, J. Vis. Commun. Image Repres., 2, 151-165, June 1991.

[14] Brockett, R.W. and Maragos, P., Evolution equations for continuous-scale morphological filtering, IEEE Trans. on Signal Processing, 42, 3377-3386, Dec. 1994.

[15] Burt, P.J. and Adelson, E.H., The Laplacian pyramid as a compact image code, IEEE Trans. Commun., 31, 532-540, Apr. 1983.

[16] Butt, M.A. and Maragos, P., Comparison of multiscale morphology approaches: PDE implemented via curve evolution versus chamfer distance transform in Mathematical Morphology and its Application to Image and Signal Processing, Maragos, P., Schafer, R.W. and Butt, M.A., Eds., Kluwer Acad. Publishing, 1996.

[17] Chen, Y. and Dougherty, E.R., Gray-scale morphological granulometric texture classification, Optical Engineering, 33, 2713-2722, Aug. 1994.

[18] Coyle, E.J. and Lin, J.H., Stack filters and the mean absolute error criterion, IEEE Trans. Acoust. Speech Signal Processing, 36, 1244-1254, Aug. 1988.

[19] Dorst, L. and van der Boomgaard, R., Morphological signal processing and the slope transform, Signal Processing, 38, 79-98, July 1994.

[20] Dougherty, E.R., Optimal mean-square N-observation digital morphological filters: I. Optimal binary filters, CVGIP: Image Understanding, 55, 36-54, Jan. 1992.

[21] Fitch, J.P., Coyle, E.J. and Gallagher, Jr., N.C., Median filtering by threshold decomposition, IEEE Trans. Acoust. Speech, Signal Processing, 32, 1183-1188, Dec. 1984.

[22] Goetcherian, V., From binary to grey tone image processing using fuzzy logic concepts, Pattern Recognition, 12, 7-15, 1980.

[23] Goutsias, J. and Shonfeld, D., Morphological representation of discrete and binary images, IEEE Trans. Signal Processing, 39, 1369-1379, June 1991.

[24] Hadwiger, H., Vorlesungen über Inhalt, Oberfläche, und Isoperimetrie, Springer-Verlag, Berlin, 1957.

[25] Haralick, R.M., Zhuang, X., Lin, C. and Lee, J.S.J., The digital morphological sampling theorem, IEEE Trans. Acoust. Speech, Signal Process, 37, 2067-2090, Dec. 1989.

[26] Heijmans, H.J.A.M. and Ronse, C., The algebraic basis of mathematical morphology-part I: Dilations and erosions, Comput. Vision Graph. Image Process., 50, 245-295, 1990.

[27] Justusson, B.I., Median filtering: Statistical properties, in Two-Dimensional Digital Signal Processing II: Transforms and Median Filters, Huang, T.S., Ed., Springer-Verlag, NY, 1981.

[28] Koenderink, J.J., The structure of images, Biol. Cybern., 50, 363-370, 1984.

[29] Kresh, R. and Malah, D., Morphological reduction of skeleton redundancy, Signal Processing, 38, 143-151, 1994.

[30] Kresh, R., Morphological image representation for coding applications, Ph.D. thesis, Technion, Israel, June 1995.

[31] Loce, R.P. and Dougherty, E.R., Facilitation of optimal binary morphological filter design via structuring element libraries and design constraints, Optical Engineering, 31, 1008-1025, May 1992.

[32] Mandelbrot, B.B., The Fractal Geometry of Nature, Freeman, San Francisco, CA, 1982. 
[33] Maragos, P., A unified theory of translation-invariant systems with applications to morphological analysis and coding of images, Ph.D. thesis, Georgia Institute of Technology, Atlanta, July 1985.

[34] Maragos, P., A representation theory for morphological image and signal processing, IEEE Trans. Pattern Anal. Mach. Intellig., 11, 586-599, June 1989.

[35] Maragos, P., Pattern spectrum and multiscale shape representation, IEEE Trans. Pattern Anal. Mach. Intellig., 11, 701-716, July 1989.

[36] Maragos, P., Fractal signal analysis using mathematical morphology, in Advances in Electronics and Electron Physics, vol. 88, Hawkes, P. and Kazan, B., Eds., Academic Press, New York, 1994, 199-246.

[37] Maragos, P., Morphological systems: Slope transforms and max-min difference and differential equations, Signal Processing, 38, 57-77, July 1994.

[38] Maragos, P., Differential morphology and image processing, IEEE Trans. Image Processing, 5, 922-937, June 1996.

[39] Maragos, P. and Schafer, R.W., Morphological skeleton representation and coding of binary images, IEEE Trans. Acoust. Speech, Signal Process., 34, 1228-1244, Oct. 1986.

[40] Maragos, P. and Schafer, R.W., Morphological filters-Part I: Their set-theoretic analysis and relations to linear shift-invariant filters, IEEE Trans. Acoust. Speech, Signal Processing, 35, 1153-1169, Aug. 1987.

[41] Maragos, P. and Schafer, R.W., Morphological filters-Part II: Their relations to median, orderstatistic, and stack filters, IEEE Trans. Acoust. Speech, Signal Process., 35, 1170-1184, Aug. 1987; ibid, 37, 597, Apr. 1989.

[42] Matheron, G., Random Sets and Integral Geometry, John Wiley \& Sons, NY, 1975.

[43] Meyer, F., Contrast feature extraction, in Quantitative Analysis of Microstructures in Materials Science, Biology and Medicine, Chermant, J.L., Ed., Special Issues of Practical Metallography, Riederer-Verlag, Stuttgart, 1978, 374-380.

[44] Meyer, F. and Becheur, S., Morphological segmentation, J. Vis. Commun. Image Representation, 1, 21-46, Sept. 1990.

[45] Nakagawa, Y. and Rosenfeld, A., A note on the use of local min and max operations in digital picture processing, IEEE Trans. Syst., Man, and Cybern., 8, 632-635, 1978.

[46] O'Neil, K.S. and Rhodes, W.T., Morphological transformations by hybrid optical-electronic methods, in Hybrid Image Processing, Casasent, D. and Tescher, A., Eds., Proc. SPIE, 638, 41-44, 1986.

[47] Osher, S. and Sethian, J.A., Fronts propagating with curvature-dependent speed: Algorithms based on Hamilton-Jacobi formulations, J. Comput. Physics, 79, 12-49, 1988.

[48] Pitas, I. and Venetsanopoulos, A., Morphological shape decomposition, IEEE Trans. Pattern Anal. Mach. Intellig., 12, 38-45, 1990.

[49] Preston, Jr., K. and Duff, M.J.B., Modern Cellular Automata, Plenum Press, New York, 1984.

[50] Rockafellar, R.T., Convex Analysis, Princeton University Press, Princeton, NJ, 1972.

[51] Roerdink, J.B.T.M., Mathematical morphology with non-commutative symmetry groups, in Mathematical Morphology in Image Processing, Dougherty, E.R., Ed., Marcel Dekker, New York, 1993.

[52] Ronse, C. and Heijmans, H.J.A.M., The algebraic basis of mathematical morphology-part II: Openings and closings, CVGIP: Image Understanding, 54, 74-97, 1991.

[53] Rosenfeld, A., Ed., Multiresolution Image Processing and Analysis, Springer-Verlag, New York, 1984.

[54] Rosenfeld, A. and Kak, A.C., Digital Picture Processing, vols. 1 and 2, Academic Press, New York, 1982.

[55] Salembier, P., Structuring element adaptation for morphological filters, J. Visual Commun. Image Repres., 3, 115-136, June 1992. 
[56] Salembier, P. and Serra, J., Morphological multiscale image segmentation, in Visual Communications and Image Processing'92, Maragos, P., Ed., Proc SPIE, 1818, 620-631, 1992.

[57] Sapiro, G., Kimmel, R., Shaked, D., Kimia, B. and Bruckstein, A., Implementing continuousscale morphology via curve evolution, Pattern Recognition, 26(9), 1363-1372, 1993.

[58] Serra, J., Image Analysis and Mathematical Morphology, Academic Press, New York, 1982.

[59] Serra, J., Ed., Image Analysis and Mathematical Morphology, Vol. 2: Theoretical Advances, Academic Press, New York, 1988.

[60] Schonfeld, D. and Goutsias, J., Optimal morphological pattern restoration from noisy binary images, IEEE Trans. Pattern Anal. Machine Intellig., 13, 14-29, Jan. 1991.

[61] Sternberg, S.R., Cellular computers and biomedical image processing, in Biomedical Images and Computers, Sklansky, J. and Bisconte, J.C., Eds., Springer-Verlag, Berlin, 1982.

[62] Sternberg, S.R., Grayscale Morphology, Comput. Vision Graph. Image Process., 35, 333-355, 1986.

[63] Szoplik, T., Ed., Selected Papers on Morphological Image Processing: Principles and Optoelectronic Implementations, SPIE Press, Bellingham, WA, 1996.

[64] Tukey, J.W., Exploratory Data Analysis, Addison-Wesley, Reading, MA, 1977.

[65] Tyan, S.G., Median filtering: Deterministic properties, in Two-Dimensional Digital Signal Processing II: Transforms and Median Filters, Huang, T.S., Ed., Springer-Verlag, New York, 1981.

[66] van der Boomgaard, R. and Smeulders, A., The morphological structure of images: The differential equations of morphological scale-space, IEEE Trans. Pattern Anal. Mach. Intellig., 16, 1101-1113, Nov. 1994.

[67] Verbeek, P.W. and Verwer, B.J.H., Shading from shape, the eikonal equation solved by greyweighted distance transform, Pattern Recogn. Lett., 11, 618-690, 1990.

[68] Vincent, L., Morphological algorithms, in Mathematical Morphology in Image Processing, Dougherty, E.R., Ed., Marcel Dekker, New York, 1993.

[69] Vincent, L. and Soille, P., Watersheds in digital spaces: An efficient algorithm based on immersion simulations, IEEE Trans. Pattern Anal. Mach. Intellig., 13, 583-598, June 1991.

[70] Wendt, P.D., Coyle, E.J. and Gallagher, N.C., Stack filters, IEEE Trans. Acoust., Speech, Signal Process., 34, 898-911, Aug. 1986.

[71] Wilson, S.S., Training structuring elements in morphological networks, in Mathematical Morphology in Image Processing, Dougherty, E.R., Ed., Marcel Dekker, New York, 1993.

[72] Witkin, A.P., Scale-space filtering, in Proc. Int'l. Joint Conf. Artif. Intellig., Karlsruhe, 1983. 